\title{
1 TITLE PAGE
}

2

3 Does your past define you? The role of previous visual experience in subjective reactions

4 to new affective pictures and sounds.

5

6 Fiorella Del Popolo Cristaldi ${ }^{1}$, Filippo Gambarota ${ }^{2}$, Suzanne Oosterwijk ${ }^{3,4}$

7

$8{ }^{1}$ Department of General Psychology, University of Padua, Padua, Italy

$9{ }^{2}$ Department of Developmental Psychology and Socialization, University of Padua, Padua, 10 Italy

$11{ }^{3}$ Department of Social Psychology, University of Amsterdam, Amsterdam, The Netherlands

$12{ }^{4}$ Amsterdam Brain and Cognition Centre (ABC), Amsterdam, The Netherlands

13

14 Corresponding author:

15 Fiorella Del Popolo Cristaldi

16 Department of General Psychology

17 Via Venezia 8, 35131 Padova, Italy

18 Phone: +390498276941

19 E-mail: fiorella.delpopolocristaldi@ phd.unipd.it 
ABSTRACT

According to predictive models of emotion, people use their previous experience to construct new affective predictions. We do not live in a stable world, however. Some environments are uncertain, others are not. This study investigated how experiencing certain vs. uncertain probabilistic relationships shapes subjective reactions to new affective stimuli, within and across sensory modalities. Two S1-S2 paradigms were employed as learning and test phases in two experiments. S1s were colored circles, S2s negative or neutral affective pictures or sounds. Participants $(\mathrm{N}=192,179)$ were assigned to the certain $(\mathrm{CG})$ or uncertain group (UG), and they were presented with $100 \%$ (CG) or $50 \%$ (UG) S1-S2 congruency between visual stimuli during the learning phase. During the test phase both groups were presented with a new S1-S2 paradigm with a 75\% S1-S2 congruency, and visual (Experiment

2) S2s. Participants were asked to rate the expected valence of upcoming S2s (expectancy ratings), or their experienced valence and arousal (valence and arousal ratings). In both experiments, participants in the CG reported more negative expectancy ratings after the S1s previously paired with negative stimuli, whereas no group differences emerged on valence and arousal ratings. Furthermore, when comparing the two experiments, no significant group by experiment interaction was found. Overall, and in line with predictive models, our results suggest that relying on a certain previous experience shapes subjective expectancies toward a coherent labeling of the predicted valence of future stimuli, and that this process develops similarly across sensory modalities.

41 Keywords: predictive coding, emotion, subjective experience, previous experience, S1-S2 paradigm 


\section{1) INTRODUCTION}

Past experience can influence our subjective reactions to future affective stimuli. Let us imagine a person who is celebrating their birthday, and is about to unwrap their presents. From previous parties, they know that their parents often give very nice gifts. They will therefore predict a joyful experience when they unwrap their parent's present, and they will be particularly pleased if the present meets their expectation. Their aunt, however, has previously given both terrible and awesome gifts. Thus, they will not have any reliable previous experience about the likelihood of receiving cool or awful items from their aunt. As a result, it will be difficult to predict their subjective reaction when unwrapping their present, and maybe they will be particularly upset if the gift turns out to be an old-fashioned knitted sweater. This example highlights how being exposed to previous certain vs. uncertain scenarios could affect people's expectancies and subjective reactions to new affective events.

\section{1) A predictive model of emotion}

According to a predictive model of emotion (Barrett, 2017; Seth \& Friston, 2016), people use their previous experience to construct affective predictive models (here we will refer to this process as the prediction generation stage), in order to predict incoming stimuli, and to prearrange action plans to efficiently deal with the expected situation (implementation stage). Actual environmental inputs are continuously tested against predictions: in case of a mismatch, the discrepancy is encoded as a prediction error, and used to adjust future predictions (updating stage). Predictive models are generative, in the sense that they generate predictions, and their modelling capacity generalizes across sensory modalities, contexts, and time (Friston, 2010; Shipp, 2016). They are probabilistic, which means that they encode the statistical structure of observed inputs (Clark, 2013; Friston, 2010; Shipp, 2016). They are also mostly implicit, potentially emerging into awareness only in case of a mismatch (Bar, 2007). The continuous cycle of generation-implementation-updating of (affective) predictions allows to (1) extract 
relevant information from the environment, (2) combine it with previously acquired knowledge, (3) pre-allocate resources to face all the potential future scenarios, and (4) consistently adjust future predictions (Barrett, 2017). Thus, this ability is crucial to promote survival (Barrett, 2017; Sterling, 2012). When applied to the subjective experience of emotion, Barrett (2017) proposes that the brain actively constructs meaning in the present moment by predicting and categorizing incoming stimuli and sensations based on past experience. When conceptual emotion knowledge is used to make meaning of actual inputs, the resulting prediction and the associated affective reaction can be subjectively experienced as an instance of emotion (Barrett, 2017; Barrett et al., 2014; Seth, 2013).

Since subjective experience of emotion emerges from the meaning making of prediction construction (Barrett, 2017; Barrett et al., 2007), and (affective) predictive models encode the probabilistic structure of observed inputs (Clark, 2013; Friston, 2010; Shipp, 2016), it follows that subjective experience might be crucially affected by the degrees of uncertainty experienced in the past, and thus by the degree of reliability of the derived predictive models. In fact, we do not live in a stable world. Human organisms are embedded in constantly changing multisensory environments, experiencing different types of stimuli (e.g., visual, auditory), with different degrees of uncertainty (i.e., carrying more or less reliable information). Therefore, tapping into internal models which are based on past multimodal experience is crucial to our brains in order to construct predictions in a dynamic and flexible way (Barrett, 2017; Friston, 2005; Peelen et al., 2010). Nevertheless, it is an open question to what extent the construction of affective predictions in one sensory modality actually relies on (and integrates) information in another sensory modality, previously experienced in uncertain vs. certain environments. Furthermore, it is still unclear how (un)certain previous experience affects future affective predictions at the

91 level of subjective experience. The present paper aims to answer these questions. Our first goal 92 is to examine which type of previously experienced environment (certain vs. uncertain) will 
93 lead to an intensification of subjective reactions during future predictions. Our second goal is

94 to examine whether new affective predictions develop similarly across sensory modalities.

\section{2) Manipulating previous experience}

Most studies in the extant literature have not manipulated previous experience directly, but have focused on how explicit probabilities of affective stimuli occurrence (based on the instructed meaning of a preceding cue) may impact affective processing. These studies mostly employed emotional S1-S2 paradigms in the visual modality. This paradigm implements a sequential presentation of two stimuli: the S1 (or cue) is typically a symbolic stimulus (e.g., geometric shapes, or letters), while the S2 (or target) is an emotional stimulus (e.g., affective pictures, or faces). Usually, S2 valence is manipulated at two levels, and a response is required to each S2 (e.g., emotion categorization, subjective valence and arousal ratings) (see Mercado et al., 2008 for a review on emotional S1-S2 paradigms). The S1-S2 paradigm allows to effectively investigate the three stages of affective prediction construction, with S1 processing reflecting the generation stage, the inter-stimulus interval (ISI) between S1 and S2 the implementation stage, and S2 the prediction updating (Del Popolo Cristaldi et al., 2021). Extant literature focused on the role of currently available probabilistic information on affective processing, by manipulating explicitly labeled certain (100\%) vs. uncertain (50\%) relationships between S1 and S2. Thus, in existing paradigms participants are explicitly instructed about the probabilistic ratios they will be exposed to. Such S1-S2 paradigms do not implement the role of actual previous experience in generating future predictions. Furthermore, they do not resemble more realistic everyday contexts, in which people automatically learn contingencies from their environments, and infer probabilistic information to use in their affective predictions, by means of exposure (Bar, 2007). The present study instead will utilize a novel experimental paradigm that directly manipulates previous experience in terms of uninstructed certain vs. uncertain emotional congruency between stimuli. 
The literature employing S1-S2 paradigms led to fragmentary evidence on subjective experience. Focusing on expectancy measures related to the generation-implementation stages

121 (i.e., either trial-by-trial expectancy ratings about the expected valence of the upcoming S2, or post-experiment estimation of the percentage of uncertain cues followed by a negative S2), it was consistently shown that an uncertain probabilistic ratio was associated with negativelybiased expectancies about the forthcoming S2 valence, which means an overestimation of negative S2s occurrence following uncertain S1s (Dieterich et al., 2016; Grupe \& Nitschke, 2011; Lin et al., 2018, 2020; Qiao et al., 2018; Sarinopoulos et al., 2010). Regarding the updating stage, some inconsistencies emerged when considering the modulating effects of the probabilistic information on S2-subjective ratings. Some studies found more pleasant valence ratings to certain positive as compared to uncertain positive $\mathrm{S} 2 \mathrm{~s}$, and more unpleasant ratings to certain negative as compared to uncertain negative S2s (Johnen \& Harrison, 2019; Lin et al., 2020; Lin, Jin, et al., 2015; Lin, Xiang, et al., 2015); one study reported more unpleasant ratings to uncertain negative as compared to certain negative S2s (Lin et al., 2017); and still others found no significant interactions between probabilistic information and subjective valence ratings (Bermpohl et al., 2006; Dieterich et al., 2016; Greenberg et al., 2015; Grupe \& Nitschke, 2011; Lin et al., 2018; Qiao et al., 2018). The only study assessing emotional intensity

136 (Bermpohl et al., 2006) found a stronger self-reported emotional intensity after certain as 137 compared to uncertain emotional S2s. Lastly, two studies (Lin et al., 2017; Lin, Xiang, et al., 138 2015) investigated how experiencing a certain vs. uncertain anticipation pattern (acquired through the emotional S1-S2 paradigm during an encoding phase) could influence the

140 subsequent recognition and the neural correlates of S2 processing during an unexpected $141 \mathrm{old} /$ new recognition task. At the neural level, it emerged that a certain anticipation pattern led 142 to an enhanced processing of negative S2s (Lin, Xiang, et al., 2015), while an uncertain 
143 anticipation pattern led to a reduced recognition of neutral S2s features (Lin et al., 2017).

144 However, it is still not clear if experiencing different degrees of (un)certainty during the

145 encoding phase could influence subsequent subjective reactions, since the authors did not

146 measure S2-affective ratings during the recognition phase.

147 Overall, these results are in contrast with the literature suggesting that uncertainty

148 intensifies affective experience, by pushing towards a polarization of both positive and negative

149 affect, and thus leading to increased attention and emotional engagement towards uncertain

150 events (Bar-Anan et al., 2009; Carleton, 2016a, 2016b; Einstein, 2014). Nevertheless, they can

151 be explained within the predictive framework (Barrett, 2017; Seth \& Friston, 2016). In certain

152 contexts, the brain can generate reliable affective predictions based on previously learned

153 contingencies, thus pre-activating the expected affective experience, which in turn could lead

154 to an intensification of subjective reactions. Within uncertain contexts, instead, reliable

155 affective predictions (and correspondent experience pre-activation) cannot be constructed, and

156 the brain's resources are deployed in prediction error detection and encoding, thus potentially

157 leading to dampened subjective reactions.

\section{1.4) The present study}

159 This brief literature review unveils some important gaps that the present study aims to

160 fill. First, the impact of previous experience on new affective predictions has never been

161 systematically investigated, although, according to the predictive framework (Barrett, 2017;

162 Seth \& Friston, 2016), prior experience is crucial to generate predictions and to make meaning

163 of the emotional events in the present moment. Second, subjective affective ratings in the extant

164 S1-S2 paradigms have mainly assessed the valence dimension, while arousal is under-

165 represented, despite being an equally important dimension of affective experience. Third,

166 extant S1-S2 paradigms are typically instructed: participants are explicitly informed about the

167 probabilistic ratios between S1 and S2 prior to the experiment. This could arouse concerns on 
the generalizability of results. In daily life, people are usually able to spontaneously learn contingencies from the environment, and to use them as priors for subsequent affective predictions, without any need to be instructed. And finally, fourth, cross-modality effects have never been studied within the extant literature. If affective predictions integrate multisensory

172 information, then it is possible that learned certain and uncertain probabilities of affective events in one modality generalize to another modality.

In the light of these considerations, we here propose a purposely developed online experimental design, which combines emotional S1-S2 paradigms with a learning component.

176 Our paradigm investigates the impact of (un)certain previous experience in constructing new

177 affective predictions in a new, moderately certain probabilistic context, both within and across sensory modalities. A first emotional S1-S2 paradigm was employed as a learning phase, in which prior experience was manipulated via uninstructed certain vs. uncertain S1-S2 emotional congruency. Participants were assigned to the certain or uncertain experimental group, in which they were presented with a $100 \%$ vs. $50 \%$ probabilistic ratio between visual stimuli (i.e., colored dots as S1s, and affective pictures as S2s), respectively. A second emotional S1-S2 paradigm with a fixed $75 \%$ S1-S2 probabilistic ratio, and visual (Experiment 1) or auditory (Experiment 2) affective stimuli as S2s, was then employed as a test phase. Here, we investigated the effects of previously learned probabilistic relationships on subjective affective experience in a new, moderately certain $(75 \%)$ probabilistic context. Participants were asked to rate the expected valence of upcoming S2s (expectancy ratings), or the experienced valence and arousal to S2s (valence and arousal ratings).

We performed confirmatory analyses to test three a-priori formulated hypotheses (as

190 pre-registered on the Open Science Framework - OSF; Experiment 1: https://osf.io/ef9q7/,

191 Experiment 2: https://osf.io/wcy9r/). The first hypothesis (H1) was that participants in the 192 uncertain group would show more negative expectancy ratings (Dieterich et al., 2016; Grupe 
193 \& Nitschke, 2011; Herwig et al., 2007; Qiao et al., 2018; Schumacher et al., 2015), as compared

194 to participants in the certain group. The second hypothesis (H2a) was that participants in the

195 uncertain group would show higher arousal and more unpleasant valence ratings to S2s (Bar-

196 Anan et al., 2009; Carleton, 2016a, 2016b; Einstein, 2014; Lin et al., 2017) than participants in

197 the certain group. The third hypothesis (H2b), opposed to H2a, was that participants in the

198 certain group would show higher arousal and more unpleasant valence ratings to S2s

199 (Bermpohl et al., 2006; Johnen \& Harrison, 2019; Lin et al., 2020; Lin, Jin, et al., 2015; Lin,

200 Xiang, et al., 2015), as compared to participants in the uncertain group.

201 Furthermore, if previously learned visual contingencies effectively generalize to novel

202 auditory stimuli, then we expected to find similar patterns of results in Experiment 1 (visual to

203 visual) and Experiment 2 (visual to auditory). Finally, we explored whether S1-S2 congruency

204 played a modulating role on S2-subjective reactions, by comparing valence and arousal ratings

205 of congruent trials (i.e., trials of the test phase in which the S1-S2 pairing was more likely, i.e.,

206 75\%) with ratings of incongruent trials (i.e., trials of the test phase in which S1-S2 pairing was

207 less likely, i.e., 25\%). 


\section{2) EXPERIMENT 1}

Experiment 1 investigated how being exposed to certain vs. uncertain probabilistic

211 relationships between visual stimuli might influence subjective affective experience to new

212 affective predictions in the same (i.e., visual) sensory modality.

213 We tested the pre-registered hypotheses H1, H2a, H2b (https://osf.io/ef9q7/).

\section{2.1) Methods}

\subsection{1) Participants}

We recruited 200 adult participants through the provider platform Prolific (Prolific,

217 Oxford, UK; www.prolific.co). The sample size was estimated through an a priori pre-

218 registered (https://osf.io/ef9q7/) simulation-based power analysis for generalized linear mixed-

219 effects models (GLMMs) (R package: simr; Green \& MacLeod, 2016), estimating parameters from pilot data $(\mathrm{N}=27)$. Data from 8 participants were discarded according to the following pre-registered exclusion criteria (https://osf.io/ef9q7/): scoring lower than 75\% accuracy on attention check items (see 2.1.2 below), reporting experienced technical issues in more than $25 \%$ of the experimental trials.

The final sample was 192 participants (73 males, age: $M=33.07, S D=11.46$, range $=$ 18-62). All participants gave their informed consent before starting the experiment, and were paid $£ 2.50$ for their participation. All experimental procedures were approved by the Ethical Committee for the Psychological Research of the University of Padua (protocol no. 4012), and were conducted in accordance with the Declaration of Helsinki.

\subsection{2) Stimulus material and procedure}

Stimuli and rating scales. Two emotional S1-S2 paradigms were employed as a

231 learning and a test phase, respectively. Colored red and blue $1 \mathrm{~cm}$ diameter circles were 232 employed as $\mathrm{S} 1 \mathrm{~s}$. An additional yellow $1 \mathrm{~cm}$ diameter circle was used as $\mathrm{S} 1$ in attention check 233 trials. Colored $800 \times 600$ px pictures (60 negative, 60 neutral) from Nencki Affective Picture 
234 System (NAPS) (Marchewka et al., 2014) were employed as S2s. NAPS pictures names, sorted

235 by emotional valence, are listed in Supplementary Table S1. Negative and neutral pictures did 236 not differ in terms of luminance, contrast, complexity, and color space (see Supplementary 237 Table S2). An additional $800 \times 600$ px picture, depicting a pattern of black stripes on a 238 transparent background, was used as S2 in attention check trials.

239 A Visual Analogue Scale (VAS) assessed expectancy ratings (i.e., the expected valence 240 of upcoming S2s), ranging from $0 \%$ ("I definitely expect to see a neutral picture") to $100 \%$ ("I

241 definitely expect to see a negative picture"), with $50 \%$ representing not knowing what to 242 expect. Two more VASs were used to collect valence and arousal ratings to S2s. The valence 243 scale ranged from $0 \%$ "very negative" to $100 \%$ "very positive", with $50 \%$ representing 244 "neutral". The arousal scale ranged from $0 \%$ "relaxed" to $100 \%$ "aroused", with $50 \%$ representing an intermediate level of activation.

Learning phase. Before the learning phase, participants were randomly assigned to one

247 of two experimental groups: the certain group (CG), and the uncertain group (UG). At the 248 beginning of the learning phase, participants were instructed to look at the computer screen and 249 pay attention to the relationship between S1 color and S2 valence. Furthermore, they were instructed to press the 'spacebar' as fast as they could each time they saw a yellow circle

251 (attention check trials). The instructions were followed by a practice session (4 trials) in which 252 participants received feedback on their performance to one attention check trial. Then, the 253 learning session started. Each trial was displayed on a grey background, and it began with S1, 254 presented for $250 \mathrm{msec}$. A fixed ISI of $1000 \mathrm{msec}$ followed, in which the screen remained grey, 255 then S2 was presented for 1000 msec. The inter-trial interval (ITI), in which a white fixation cross was displayed in the center of the screen, randomly varied between 800 and $1200 \mathrm{msec}$.

257 The total number of trials was 40. The order of the trials was random. 

congruency: each S1 color was paired with the same S2 valence in $100 \%$ of the trials, thus they learned a highly predictive relationship between S1 and S2. The UG, instead, experienced a $50 \%$ congruency level: each S1 color was paired with negative S2s in $50 \%$ of the trials, and with neutral S2s in the other 50\%, thus they learned an uncertain relationship. Color-valence pairings were counterbalanced between subjects. Participants were left uninstructed about the S1-S2 probabilistic relationship.

Test phase. At the end of the learning phase participants were asked to wait and relax for 1 minute, after which they were introduced to the test phase, identical for both the experimental groups. At the beginning of the test phase participants were instructed about the second S1-S2 paradigm: they were asked to look at the computer screen and pay attention to S1 color, trying to predict S2 emotional valence. Participants were explained that, in some trials, they would be asked to rate how much they expected to see a negative picture after the S1, by answering a $0-100 \%$ rating scale; while in other trials they would be asked to assess the subjective valence and arousal elicited by the S2, by answering a 0-100\% rating scale for each dimension. They were also instructed to press the 'spacebar' as fast as they could each time they saw the yellow circle (attention check). Instructions were followed by a practice session (4 trials) in which participants trained themselves to give their ratings, and they received feedback on their performance to one attention check trial. Then, the test session started. Trial structure and timing were the same as the learning phase. The total number of trials was 80 .

278 S1-S2 congruency was fixed at $75 \%$, that is S1 color was moderately predictive of S2 valence 279 (same S1 color-S2 valence pairings in $75 \%$ of the trials). Color-valence pairings were counterbalanced between subjects. Participants were left uninstructed about the S1-S2 probabilistic relationship in the test phase. 

(expectancy ratings), or right after the $\mathrm{S} 2$ for the other half of the trials (valence and arousal ratings). The order of the trials was random. Response time for the VASs was self-paced. The expectancy ratings and the valencelarousal ratings were presented in different trials to prevent the two ratings from influencing each other (see Figure 1a for a schematic representation of the experimental paradigm).

Congruency. In the test phase, trials in which the pairing between S1 color and S2

valence was more likely (i.e., 75\%) were coded as congruent, while trials in which the pairing between S1 color and S2 valence was less likely (i.e., 25\%) were coded as incongruent. Thus, congruent trials are those trials in which, according to the new $75 \%$ probabilistic ratio, a stronger expectancy about the valence of the forthcoming S2 should be generated and implemented, leading to an expectancy confirmation when updating the internal model to the actual S2. In incongruent trials, instead, the lower likelihood of the S1-S2 association should determine an expectancy violation when updating the internal model to the actual S2. Therefore, the $S 2$ congruency factor allowed us to investigate if ratings were different between expected and unexpected S2s, combining the new contingencies with the contingencies learned in the learning phase. Notably, the $75 \%$ ratio implied participants in the CG to transition from a totally reliable predictive context (100\%, experienced during the learning phase), to a new context in which the predictive models they had previously learned were confirmed in the congruent trials and disconfirmed in the incongruent trials. Participants in the UG, instead, transitioned from a totally unreliable predictive context (50\%), to a more predictive situation. Importantly, the $75 \%$ ratio is equidistant from the probabilistic relationships experienced both by the CG and the UG, laying exactly in the middle between $100 \%$ and $50 \%$. and the JATOS hosting server (Lange et al., 2015). Participants were instructed to ensure that 
307 their environment was optimal for participation: they were asked to sit alone in a silent and

308 private room, and to avoid all potential distractions and interruptions. Furthermore, they were

309 asked to perform the study on a computer, and to ensure that no one else could view the

310 computer screen during the experiment due to the involvement of emotionally evocative

311 material.

312 At the end of the test phase, participants were redirected to a Qualtrics survey

313 (Qualtrics, Provo, UT; www.qualtrics.com). They were asked to fill a few demographic

314 questions (age, gender). They were also asked whether and in how many trials they had

315 experienced any issues with the internet connection and/or with pictures uploading. At the end

316 of the survey, all participants were thanked for their participation, and redirected to Prolific to

317 complete the study submission and receive their payment. 
a) EXPERIMENT 1

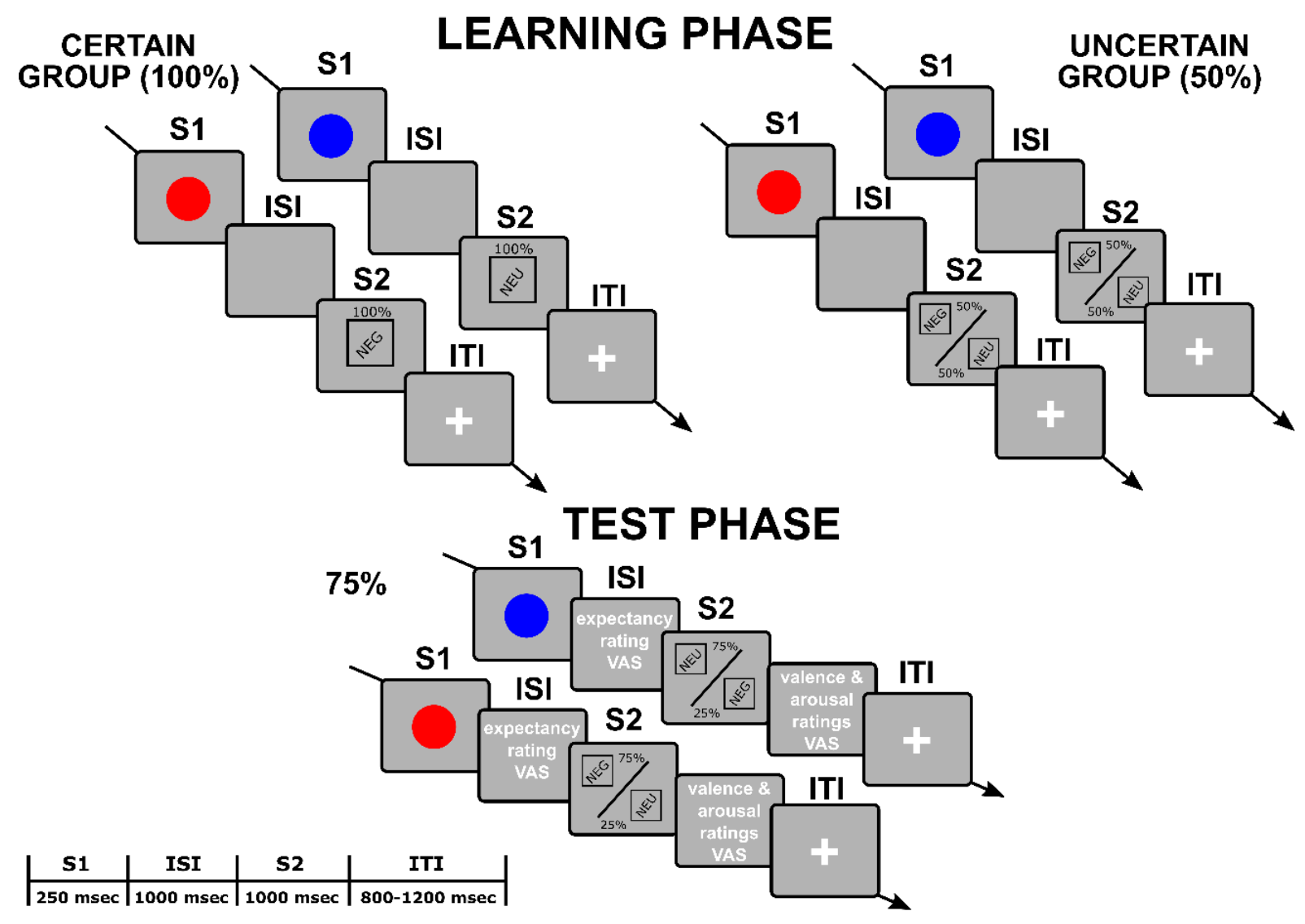

b) EXPERIMENT 2

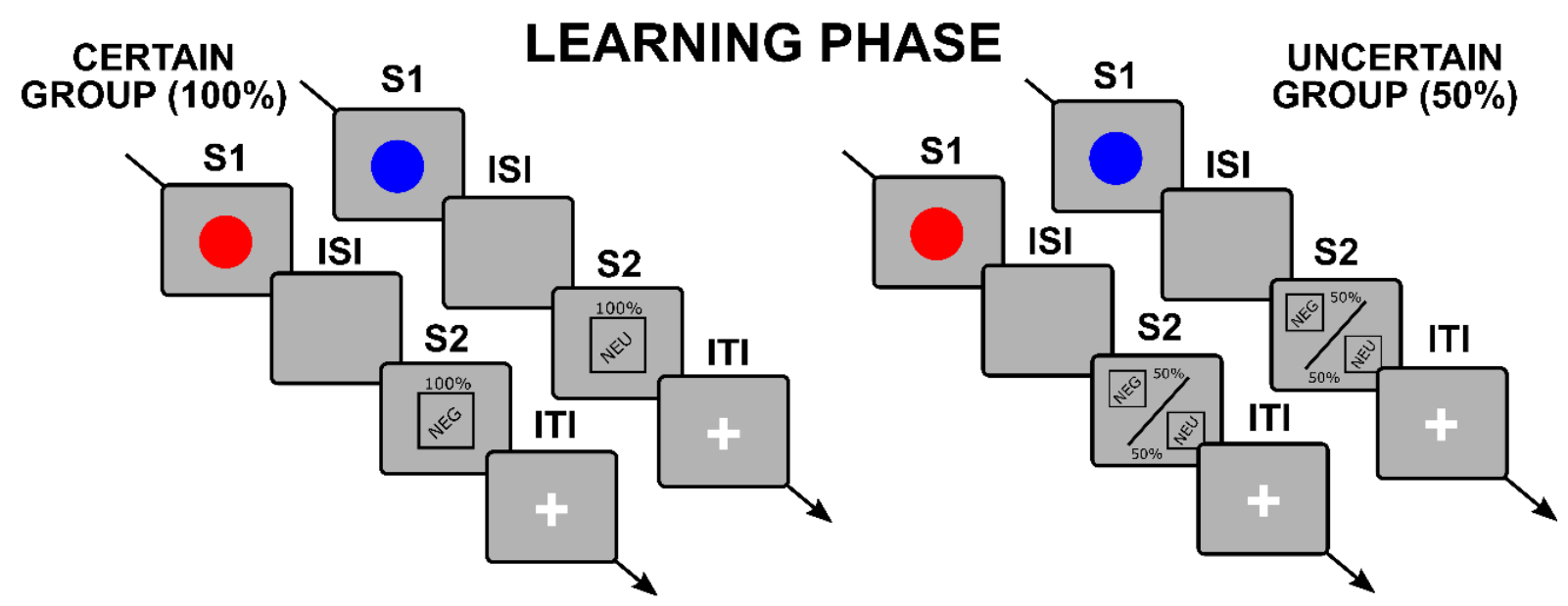

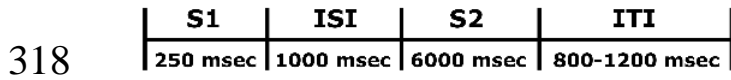


319 Figure 1. a) Example sequence of events and their duration for a trial of Experiment 1, 320 according to the phase (learning, test), and the experimental group (CG, UG). During the

321 learning phase participants experienced a 100\% (CG) vs. 50\% (UG) probabilistic relationship 322 between S1s (red or blue circles) and S2s (negative or neutral emotional pictures), according 323 to the experimental group. During the test phase the S1-S2 probabilistic relationship was fixed at $75 \%$ for both groups. Participants were asked to answer the VASs either during the ISI for half of the trials (expectancy ratings), or right after the $\mathrm{S} 2$ for the other half of the trials (valence and arousal ratings). Response times were self-paced. b) Example sequence of events and their duration for a trial of Experiment 2, according to the phase (learning, test), and the experimental group (CG, UG). During the learning phase participants experienced a $100 \%$ (CG) vs 50\% (UG) probabilistic relationship between S1s (red or blue circles) and S2s (negative or neutral affective pictures), according to the experimental group. During the test phase S2s were negative or neutral affective sounds, and the S1-S2 probabilistic relationship was fixed at $75 \%$. Participants were asked to answer the VASs either during the ISI for half of the trials (expectancy ratings), or right after the $\mathrm{S} 2$ for the other half of the trials (valence and arousal ratings). Response times were self-paced.

ISI $=$ inter-stimulus interval, ITI = inter-trial interval, VAS $=$ visual analogue scale. The text is not drawn to scale.

\subsection{3) Data analysis}

The study has a 2 (group, between-subjects: CG vs. UG) $\times 2$ (S2 valence, within-

340 subjects: negative - neg vs. neutral - neu) mixed design. The analysis plan was pre-registered on OSF (https://osf.io/ef9q7/). We ran an additional analysis to investigate if expectancy

342 ratings in the two groups differed as a function of the $S 1$ color (within-subjects: red vs. blue).

343 In fact, based on the way the paradigm is constructed, the color of the circle had a different 
344 (uninstructed) predictive value depending on the experimental group. Each color had an actual

345 predictive meaning for participants in the CG, since they experienced a totally reliable S1-S2

346 congruency ratio during the learning phase (for example, they learned that a red circle always

347 preceded a negative picture, and a blue one always preceded a neutral picture). For participants

348 in the UG, instead, the color of the circle had no predictive value, since during the learning

349 phase each color was paired equally often with either negative or neutral pictures. To

350 compensate for the counterbalancing of S1 color-S2 valence pairings, in the analysis we re-

351 coded as red all the trials in which the circle preceded a negative picture, and as blue all the

352 trials in which the circle preceded a neutral picture, independently from the actual circles' color.

353 Outliers were detected through Median Absolute Deviation values (MAD > 3) when

354 univariate (i.e., expectancy ratings), and through the Mahalanobis-Minimum Covariance

355 Determinant (MMCD, breakdown point 0.25) when multivariate (i.e., valence and arousal

356 ratings) (R package: Routliers; Leys et al., 2019). Data from 7 participants were detected as

357 univariate outliers. From visual inspection of their ratings, it emerged that these participants

358 had reversed the poles of the rating scale ("error outliers", cf. Leys et al., 2019), thus they were

359 removed from data analysis. Data from 68 participants were detected as multivariate outliers.

360 From visual inspection of their ratings, it emerged that these participants were characterized by

361 a slightly flattened or steeper relationship between valence and arousal ratings as compared to

362 other participants. Thus, since none of them significantly impacted the models' estimates (as

363 assessed through Cook's distance, see below), we chose not to discard them ("potentially

364 interesting outliers", cf. Leys et al., 2019). Data from 185 participants entered data analysis.

365 In order to test our a-priori hypotheses $(\mathrm{H} 1, \mathrm{H} 2 \mathrm{a}, \mathrm{H} 2 \mathrm{~b})$, for each dependent variable

366 (DV) we fitted the following linear mixed-effects models (LMMs) (R package: lme4; Bates et

367 al., 2015): 
1. expectancy ratings (H1): group, $\mathrm{S} 1$ color and their interaction as fixed factors, random slopes for S1 color within participant;

2. valence and arousal ratings ( $\mathrm{H} 2 \mathrm{a}$ vs. $\mathrm{H} 2 \mathrm{~b})$ : group, $\mathrm{S} 2$ valence and their interaction as fixed factors, random slopes for $S 2$ valence within participant.

Furthermore, to assess the differences between expected and unexpected S2s, we ran the following exploratory analysis: we added S2 congruency (within-subjects: congruent vs. incongruent), and its interaction with group and $S 2$ valence as fixed factors to the confirmatory models for valence and arousal ratings. calculated via Satterthwaite's degrees of freedom method $(\alpha=.05, \mathrm{R}$ package: 1merTest; Kuznetsova et al., 2017). For each model we reported the estimated parameters with 95\% Nakagawa et al., 2017), that represent the variance explained by fixed effects and by fixed plus random effects, respectively.

\subsection{4) Transparency and openness}

The study has been pre-registered on OSF (https://osf.io/ef9q7/). The experiment's file (containing all the stimulus material), the pilot pre-processed dataset, and the power analysis $\mathrm{R}$ syntax are included in the pre-registration. The final pre-processed dataset, and the analysis $\mathrm{R}$ syntax are available at the GitHub repository linked to the pre-registration

388 (https://osf.io/ef9q7/).

\section{2) Results}

All confirmatory models are summarized in Table 1 and Figure 2. For the expectancy

391 model $\left(\mathrm{R}^{2}\right.$ marginal $=0.216, \mathrm{R}^{2}$ conditional $\left.=0.496\right)$, we found a main effect of $S 1$ color $(F(1,183)=$ $39290.2, p<0.001)$ and an interaction between $S 1$ color and $\operatorname{group}(F(1,183)=65.30 p<0.001)$. 
393 In line with the assumption that participants learned probabilistic relationships in the learning 394 phase, we found that participants in the CG reported significantly more negative expectancy 395 ratings after the red color (i.e., circles preceding negative pictures) as compared to the blue 396 color (i.e., circles preceding neutral pictures) (blue - red $=-35.2, t(183)=-13.12, p<0.001$, $39795 \% \mathrm{CI}=[-40.54,-29.94])$, whereas in the UG no differences in expectancy ratings as a 398 function of the $\mathrm{S} 1$ color emerged (blue - red $=-2.8, t(183)=-0.96, p=0.34,95 \% \mathrm{CI}=[-8.72$, 399 3.03]). Contrary to H1, however, we did not find any significant main effect of group ( $F(1$, $400183)=0.02, p=0.9$ ). Thus, the expectancy model does not support the hypothesis of more 401 negative expectancy ratings in the UG as compared to the CG (H1).

402 For both valence $\left(\mathrm{R}^{2}\right.$ marginal $=0.622, \mathrm{R}^{2}$ conditional $\left.=0.723\right)$ and $\operatorname{arousal}\left(\mathrm{R}^{2}\right.$ marginal $=0.293$, $403 \mathrm{R}_{\text {conditional }}^{2}=0.556$ ) models testing $\mathrm{H} 2 \mathrm{a}$ vs. $\mathrm{H} 2 \mathrm{~b}$, we found a main effect of $S 2$ valence, with 404 both groups reporting significantly greater unpleasantness $(F(1,183)=1393, p<0.001$, neg 405 neu $=-47.55, \mathrm{SE}=1.27, t(183)=-37.32, p<0.001,95 \% \mathrm{CI}=[-50.06,-45.03])$ and higher arousal $(F(1,183)=332.68, p<0.001$, neg - neu $=26.69, \mathrm{SE}=1.46, t(183)=18.24, p<0.001$, $40795 \% \mathrm{CI}=[23.79,29.57])$ towards negative pictures. However, in contrast with $\mathrm{H} 2 \mathrm{a}$ and $\mathrm{H} 2 \mathrm{~b}$, 408 we found no evidence for the interaction effect between $S 2$ valence and group both for valence $409(F(1,183)=0.07, p=0.799)$ and arousal ratings $(F(1,183)=0.82, p=0.364)$. Thus, neither 410 valence nor arousal models support the hypothesis of an affect intensification in the UG (H2a) 411 or in the $\mathrm{CG}(\mathrm{H} 2 \mathrm{~b})$ as compared to the other group.

412 Exploratory models are summarized in Table 2. 


\begin{tabular}{|c|c|c|c|c|c|c|c|c|}
\hline Model & Parameter & Estimate & SE & $t$ & df & $p$ & $95 \%$ & CI \\
\hline \multirow{7}{*}{ Expectancy ratings } & Intercept & 52.96 & 0.57 & 93.46 & 183.00 & $<0.001$ & 51.84 & 54.08 \\
\hline & $\mathrm{UG}-\mathrm{CG}$ & -0.15 & 1.13 & -0.13 & 183.00 & 0.897 & -2.38 & 2.09 \\
\hline & blue - red & -19.04 & 2.00 & -9.50 & 182.99 & $<0.001$ & -23.00 & -15.09 \\
\hline & color x group & -32.40 & 4.01 & -8.08 & 182.99 & $<0.001$ & -40.31 & -24.49 \\
\hline & $\sigma$ ID & 6.98 & & & & & & \\
\hline & $\sigma$ color & 26.37 & & & & & & \\
\hline & $\sigma$ residual & 20.04 & & & & & & \\
\hline \multirow{7}{*}{ Valence ratings } & Intercept & 45.49 & 0.40 & 112.42 & 183.00 & $<0.001$ & 44.69 & 46.28 \\
\hline & $\mathrm{UG}-\mathrm{CG}$ & -0.73 & 0.81 & -0.90 & 183.00 & 0.369 & -2.33 & 0.87 \\
\hline & neg - neu & -47.55 & 1.27 & -37.32 & 183.00 & $<0.001$ & -50.06 & -45.04 \\
\hline & valence $\mathrm{x}$ group & 0.65 & 2.55 & 0.26 & 183.00 & 0.799 & -4.38 & 5.68 \\
\hline & $\sigma$ ID & 4.87 & & & & & & \\
\hline & $\sigma$ valence & 16.49 & & & & & & \\
\hline & $\sigma$ residual & 15.87 & & & & & & \\
\hline \multirow{6}{*}{ Arousal ratings } & Intercept & 47.67 & 0.63 & 75.12 & 183.00 & $<0.001$ & 46.42 & 48.93 \\
\hline & $\mathrm{UG}-\mathrm{CG}$ & 0.17 & 1.27 & 0.13 & 183.00 & 0.894 & -2.33 & 2.67 \\
\hline & neg - neu & 26.69 & 1.46 & 18.24 & 183.00 & $<0.001$ & 23.80 & 29.57 \\
\hline & valence $\mathrm{x}$ group & -2.66 & 2.93 & -0.91 & 183.00 & 0.364 & -8.44 & 3.11 \\
\hline & $\sigma$ ID & 8.19 & & & & & & \\
\hline & $\sigma$ valence & 19.11 & & & & & & \\
\hline
\end{tabular}


Table 1. Results of confirmatory LMMs on expectancy, valence and arousal ratings in

415 Experiment 1. For each model, we reported the unstandardized regression coefficients, 416 standard errors (SE), 95\% confidence intervals (CI), and the associated $t$-test.

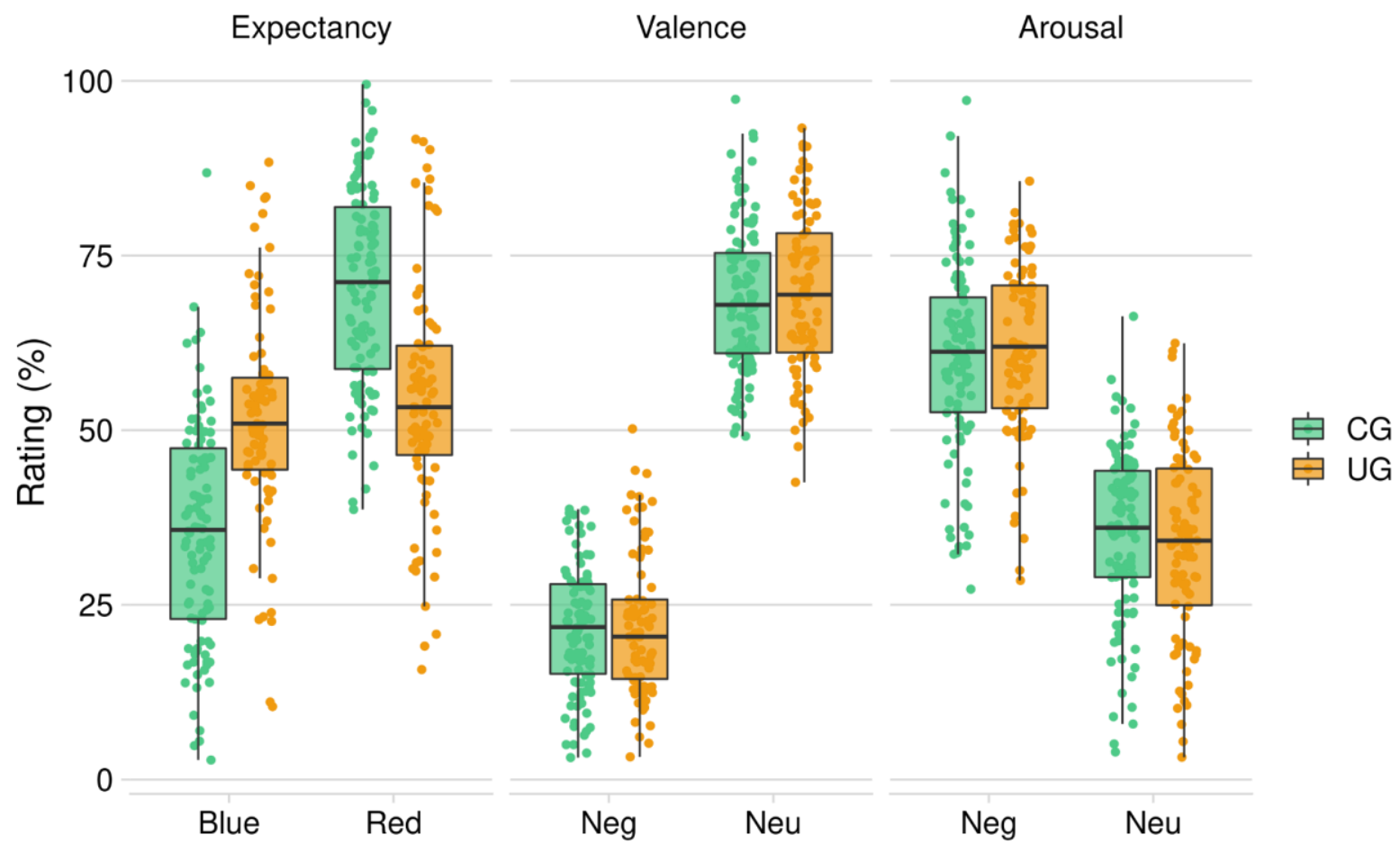

418 Figure 2. Box-plot of expectancy, valence and arousal ratings in Experiment 1 according to

419 the group (CG vs. UG), and the S1 color (red vs. blue, for expectancy ratings) or the S2 valence 420 (neg vs. neu, for valence and arousal ratings). Points represent the mean estimated value for 421 each participant and condition. 


\begin{tabular}{|c|c|c|c|c|c|c|c|c|}
\hline Model & Parameter & Estimate & SE & $t$ & df & $p$ & 950 & CI \\
\hline \multirow{11}{*}{$\begin{array}{l}\text { Valence } \\
\text { ratings }\end{array}$} & Intercept & 46.44 & 0.42 & 111.05 & 208.84 & $\begin{array}{c}< \\
0.001\end{array}$ & 45.62 & 47.27 \\
\hline & $\mathrm{UG}-\mathrm{CG}$ & -0.52 & 0.84 & -0.62 & 208.84 & 0.535 & -2.17 & 1.13 \\
\hline & neg - neu & -45.60 & 1.29 & -35.30 & 193.21 & $\begin{array}{c}< \\
0.001\end{array}$ & $\begin{array}{c}- \\
48.14\end{array}$ & 43.05 \\
\hline & congruent - incongruent & -3.83 & 0.42 & -9.06 & $7,026.01$ & $\begin{array}{c}< \\
0.001\end{array}$ & -4.66 & -3.00 \\
\hline & valence $\mathrm{x}$ group & 1.10 & 2.58 & 0.43 & 193.21 & 0.669 & -3.99 & 6.20 \\
\hline & group $\mathrm{x}$ congruency & -0.84 & 0.85 & -0.99 & $7,026.01$ & 0.322 & -2.50 & 0.82 \\
\hline & valence $\mathrm{x}$ congruency & -7.82 & 0.85 & -9.24 & $7,026.01$ & $\begin{array}{c}< \\
0.001\end{array}$ & -9.47 & -6.16 \\
\hline & $\begin{array}{l}\text { group x valence } \mathrm{x} \\
\text { congruency }\end{array}$ & -1.82 & 1.69 & -1.07 & $7,026.01$ & 0.283 & -5.14 & 1.50 \\
\hline & $\sigma$ ID & 4.88 & & & & & & \\
\hline & $\sigma$ valence & 16.51 & & & & & & \\
\hline & $\sigma$ residual & 15.68 & & & & & & \\
\hline \multirow{8}{*}{$\begin{array}{l}\text { Arousal } \\
\text { ratings }\end{array}$} & Intercept & 47.33 & 0.64 & 73.48 & 194.20 & $\begin{array}{c}< \\
0.001\end{array}$ & 46.06 & 48.60 \\
\hline & $\mathrm{UG}-\mathrm{CG}$ & 0.17 & 1.29 & 0.13 & 194.20 & 0.893 & -2.37 & 2.71 \\
\hline & neg - neu & 25.53 & 1.48 & 17.26 & 191.40 & $\begin{array}{c}< \\
0.001\end{array}$ & 22.62 & 28.45 \\
\hline & congruent - incongruent & 1.36 & 0.44 & 3.09 & $7,026.00$ & 0.002 & 0.50 & 2.23 \\
\hline & valence $\mathrm{x}$ group & -2.47 & 2.96 & -0.83 & 191.40 & 0.405 & -8.31 & 3.37 \\
\hline & group $\mathrm{x}$ congruency & -0.01 & 0.88 & -0.02 & $7,026.00$ & 0.987 & -1.74 & 1.71 \\
\hline & valence $\mathrm{x}$ congruency & 4.61 & 0.88 & 5.22 & $7,026.00$ & $\begin{array}{c}< \\
0.001\end{array}$ & 2.88 & 6.33 \\
\hline & $\begin{array}{l}\text { group x valence } \mathrm{x} \\
\text { congruency }\end{array}$ & -0.77 & 1.76 & -0.44 & $7,026.00$ & 0.663 & -4.22 & 2.69 \\
\hline
\end{tabular}




\begin{tabular}{l}
$\frac{\sigma \text { ID }}{\sigma \text { valence }} 19.11$ \\
\hline$\sigma$ residual \\
\hline sults of exploratory LMMs investigating the effect of S2 congruency (congruent \\
rent) on valence and arousal ratings in Experiment 1. For each model, we reported \\
ciated $t$-test.
\end{tabular}

\section{3) Discussion}

Experiment 1 demonstrated that within the visual sensory modality previous

430 probabilistic information shaped the subjective experience of the expected valence of future

431 stimuli (i.e., the generation-implementation stages of affective prediction construction), but not

432 of their experienced valence and intensity (i.e., the updating stage). Participants in the CG -

433 who during the learning phase were exposed to totally reliable S1-S2 relationships - during the

434 test phase reported more negative expectancy ratings after the cues whose color was previously

435 paired with negative pictures; whereas expectancy ratings of participants in the UG were not

436 modulated by the color of the cue. This suggests that, within the visual modality, experiencing

437 a past environment conveying certain probabilistic information leads people's subjective

438 expectancies to subsequently rely on previous experience to consistently generate and

439 implement new affective predictions. Having learned that a specific environmental cue reliably

440 predicts a negative stimulus will thus coherently modulate subjective expectancies towards a

441 more negative valence. Contrary to our hypothesis (H1), however, we did not find any evidence

442 of negatively-biased expectancy ratings in the UG. This unexpected result can be explained by

443 the differences between our experimental paradigm and the extant literature from which we

444 derived our hypothesis (Dieterich et al., 2016; Grupe \& Nitschke, 2011; Herwig et al., 2007; 
445 Qiao et al., 2018; Schumacher et al., 2015). The latter measured expectancy in relation to 446 currently available, instructed probabilistic information; whereas in our experimental 447 paradigm participants previously learned uninstructed certain or uncertain contingencies, and 448 only in a subsequent moment they were asked to express their expectancy ratings within a 449 different probabilistic context. It is possible that experiencing a high uncertainty level (i.e., $45050 \%$ ) in the here and now might push subjective experience towards a negative bias (as it has been found in the literature), whereas this effect might be dampened when uncertain past experience is used at a later time to generate and implement new predictions (as it happens in our paradigm).

Regarding the updating stage, we found no evidence for an affect intensification effect strictly related to previous experience. In fact, no significant group differences emerged on valence and arousal ratings. This result is in contrast with our hypotheses ( $\mathrm{H} 2 \mathrm{a}$ and $\mathrm{H} 2 \mathrm{~b})$, which were derived from the literature assessing subjective experience with regard to currently available, instructed probabilistic information (Johnen \& Harrison, 2019; Lin et al., 2017, 2020; Lin, Jin, et al., 2015; Lin, Xiang, et al., 2015). It is however consistent with some extant evidence of null interactions between probabilistic information and subjective valence ratings (Bermpohl et al., 2006; Dieterich et al., 2016; Greenberg et al., 2015; Grupe \& Nitschke, 2011; Lin et al., 2018; Qiao et al., 2018). Thus, subjective reactions during the updating stage do not seem to be affected by previous (un)certain learning, showing only a valence-dependent modulation, with both groups reporting greater unpleasantness and higher arousal towards negative stimuli. This can be partially ascribed to a ceiling effect, such that the intrinsic differences in valence between negative and neutral visual stimuli were so prominent that they might have eventually captured most of the variance in the valence and arousal ratings. Thus, given that results of Experiment 1 suggested that previous experience shaped the 469 subjective reactions to new affective predictions only with regards to the generation- 
471 stage, in Experiment 2 we tested if these results hold when previous experience and new 472 predictions tap into different sensory modalities.

473 


\section{3) EXPERIMENT 2}

475

476

477

478

479

480

481

482

483

484

485

486

487

488

489

490

491

492

493

494

495

496

497

498

Experiment 2 investigated how being exposed to certain vs. uncertain probabilistic relationships between stimuli in one sensory modality (i.e., visual) might influence the subjective affective experience to new affective predictions when it crosses over to a different sensory modality (i.e., auditory).

We tested the same a-priori and pre-registered (https://osf.io/wcy9r/) hypotheses as in Experiment 1 (H1, H2a, H2b). Furthermore, we explored whether the influence of previous experience on subjective affective ratings differed between Experiment 1 and Experiment 2.

\section{1) Methods}

\subsection{1) Participants}

According to an a-priori power-analysis (see 2.1.1 for details), we recruited 200 adult participants through the provider platform Prolific (Prolific, Oxford, UK; www.prolific.co).

Data from 17 participants were discarded according to the pre-registered exclusion criteria (https://osf.io/wcy9r/). Furthermore, data from 3 participants were discarded because of data collection failure, and 1 participant reported to be affected by hearing impairment, thus they were excluded from data analysis.

The final sample was 179 participants (97 males, age: $M=26.05, S D=7.86$, range $=$ 18-59). All participants gave their informed consent before starting the experiment, and were paid $£ 2.67$ for their participation. All experimental procedures were approved by the Ethical Committee for the Psychological Research of the University of Padua (protocol no. 4012), and were conducted in accordance with the Declaration of Helsinki.

\subsection{2) Stimulus material and procedure}

The experiment was run online, through OpenSesame (Mathôt et al., 2012) and the JATOS hosting server (Lange et al., 2015). Two S1-S2 paradigms were employed as a learning and a test phase, respectively. S1s were the same as in Experiment 1. NAPS pictures (20 

negative, 20 neutral) (Marchewka et al., 2014) were employed as S2s for the learning phase.

500 Affective sounds (40 negative, 40 neutral) from International Affective Digitized Sounds 501 (IADS-2) (Bradley \& Lang, 2007) were employed as S2s for the test phase. NAPS pictures 502 names, and IADS-2 sounds numbers, sorted by valence, are listed in Supplementary Table S3. 503 Negative and neutral pictures, and sounds did not differ in terms of physical properties (i.e., 504 luminance, contrast, complexity, and color space for pictures; max $\mathrm{dB}$, min $\mathrm{dB}$, and peak $\mathrm{dB}$ for sounds) (see Supplementary Table S4). During attention check trials, a white noise sound was used as S2 for the test phase. Three VASs were used to collect expectancy (from $0 \%$ "I definitely expect to hear a neutral sound" to $100 \%$ "I definitely expect to hear a negative sound"), valence (from $0 \%$ "very negative" to 100\% "very positive") and arousal (from 0\% "relaxed" to $100 \%$ "aroused") ratings.

Before the learning phase participants were randomly assigned to the CG or the UG.

511 They were instructed to ensure silent and private environmental conditions for participation:

512 they were asked to avoid potential distractions and interruptions, and to perform the study 513 alone, where no one else could view the computer screen nor hear the sounds due to the 514 involvement of emotionally evocative material. The learning phase was identical to Experiment 5151.

At the end of the learning phase participants were asked to wait and relax for 1 minute,

517 and then they were introduced to the test phase, identical for both groups. Instructions, practice 518 trials, and trial number and structure of the test phase were the same as in Experiment 1 519 (adapting the wording from visual to auditory modality). S1, ISI, and ITI timings were unchanged, whereas S2s were presented in their full length of $6000 \mathrm{msec}$ (see Figure $1 \mathrm{~b}$ for a schematic representation of the experimental paradigm).

At the end of the test phase, participants were redirected to the Qualtrics survey (see 2.1.2), and then back to Prolific to complete the study submission and receive their payment. 


\subsection{3) Data analysis}

The study has a 2 (group, between-subjects: CG vs. UG) $\times 2$ (S2 valence, withinsubjects: negative - neg vs. neutral - neu) mixed design. The analysis plan was pre-registered on OSF (https://osf.io/wcy9r/).

Data from 11 participants were detected as univariate outliers (MAD > 3). From visual inspection of their ratings, it emerged that 7 of them had reversed the poles of the rating scale

530 ("error outliers", cf. Leys et al., 2019), thus they were excluded from data analysis. Data from 54 participants were detected as multivariate outliers (MMCD, breakdown point 0.25). From visual inspection of their ratings, 1 of them was identified as having reversed the rating scales' poles (“error outlier", cf. Leys et al., 2019), and removed from data analysis. The remaining 57 outliers were characterized by a slightly flattened or steeper relationship between valence and arousal ratings as compared to other participants. Thus, since none of them significantly impacted the models' estimates (as assessed through Cook's distance), we chose to keep them into data analysis ("potentially interesting outliers", cf. Leys et al., 2019) . Data from 171 participants entered data analysis.

In order to test our a-priori hypotheses $(\mathrm{H} 1, \mathrm{H} 2 \mathrm{a}, \mathrm{H} 2 \mathrm{~b})$, for each $\mathrm{DV}$ we fitted the following LMMs (R package: lme4; Bates et al., 2015):

1. expectancy ratings $(\mathrm{H} 1)$ : group, $S 1$ color and their interaction as fixed factors, random slopes for S1 color within participant;

2. valence and arousal ratings ( $\mathrm{H} 2 \mathrm{a}$ vs. $\mathrm{H} 2 \mathrm{~b})$ : group, $\mathrm{S} 2$ valence and their interaction as fixed factors, random slopes for $S 2$ valence within participant. Furthermore, we ran some exploratory analyses. To assess the differences between

547 valence as fixed factors to the confirmatory models for valence and arousal ratings. To compare results from Experiment 1 and Experiment 2, we merged the two datasets, and added 
549 the study (between-subjects: Experiment 1 vs. Experiment 2) as a fixed factor to the

550

551

552

553

554

555

556

557

558

559

560

561

562

563

564

565

566

567

568

569

570

571

572

573 confirmatory LMMs.

For each confirmatory model no influential cases emerged, as evaluated through Cook's distance (>1). Models effects were evaluated using $F$-test and $p$-values, calculated via Satterthwaite's degrees of freedom method ( $\alpha=.05$, R package: lmerTest; Kuznetsova et al., 2017). For each model we reported the estimated parameters with $95 \%$ CI. We also reported marginal and conditional $\mathrm{R}^{2}$ (estimated as in Nakagawa et al., 2017), that represent the variance explained by fixed effects and by fixed plus random effects, respectively.

\subsection{4) Transparency and openness}

The study has been pre-registered on OSF (https://osf.io/wcy9r/). The experiment's file (containing all the stimulus material) is included in the pre-registration. The final pre-processed dataset, and the analysis $\mathrm{R}$ syntax are available at the GitHub repository linked to the preregistration (https://osf.io/wcy9r/).

\section{2) Results}

All confirmatory models are summarized in Table 3 and Figure 3. For the expectancy model $\left(\mathrm{R}^{2}\right.$ marginal $\left.=0.145, \mathrm{R}_{\text {conditional }}^{2} 0.468\right)$ replicating the finding from Experiment 1 , we found a main effect of $S 1$ color $(F(1,183)=73.25, p<0.001)$, and an interaction between $S 1$ color and group $(F(1,183)=18.45, p<0.001)$. In particular, we found that participants reported significantly more negative expectancy ratings after the red color (i.e., circles preceding negative sounds) as compared to the blue one (i.e., circles preceding neutral sounds), both in the CG (blue - red $=-27.97, t(183)=-8.86, p<0.001,95 \% \mathrm{CI}=[-34.20,-21.73])$ and in the UG (blue - red $=-9.28, t(183)=-3.09, p=0.002,95 \% \mathrm{CI}=[-15.19,-3.36])$ condition. To better understand these two contrasts we computed a standardized effect size, dividing the mean difference between blue and red cues within each group by the total estimated variability from the LMM (as in Westfall et al., 2014). The blue vs. red standardized difference was -0.81 
in the CG and -0.269 in the UG. Thus, despite both contrasts being statistically significant, the

575

576

577

578

579

580

581

582

583

584

585

586

587

588

589

590

591

592

593

594

595

standardized difference between red and blue cues is clearly greater (and therefore of relevant interest for the purposes of an interpretation) in the CG. Contrary to H1, we did not find any significant main effect of $\operatorname{group}(F(1,183)=0.35, p=0.56)$. Therefore, the expectancy model does not support the hypothesis of more negative expectancy ratings in the UG as compared to the $\mathrm{CG}(\mathrm{H} 1)$.

For both valence $\left(\mathrm{R}^{2}\right.$ marginal $\left.=0.471, \mathrm{R}_{\text {conditional }}^{2}=0.540\right)$ and $\operatorname{arousal}\left(\mathrm{R}^{2}\right.$ marginal $=0.274$, $\mathrm{R}_{\text {conditional }}=0.398$ ) models testing $\mathrm{H} 2 \mathrm{a}$ vs. $\mathrm{H} 2 \mathrm{~b}$, we found a main effect of $S 2$ valence, with both groups reporting significantly greater unpleasantness $(F(1,169)=1490.65, p<0.001$, neg - neu $=-37.67, \mathrm{SE}=0.98, t(183)=-38.61, p<0.001,95 \% \mathrm{CI}=[-39.59,-35.74])$ and higher $\operatorname{arousal}(F(1,169)=870.40, p<0.001$, neg - neu $=25.24 \mathrm{SE}=0.86, t(183)=-37.32, p<0.001$, $95 \% \mathrm{CI}=[23.55,26.93])$ towards negative sounds. We also found evidence for interaction between $S 2$ valence and group on both valence $(F(1,169)=4.72, p=0.031)$ and arousal models $(F(1,169)=5.30, p=0.022)$, as hypothesized $(\mathrm{H} 2 \mathrm{a}$ and $\mathrm{H} 2 \mathrm{~b})$. However from post-hoc contrasts we did not find evidence for significant differences between the CG and the UG within each $S 2$ valence level for both valence (neu: $\mathrm{CG}-\mathrm{UG}=2.54, \mathrm{SE}=1.32, t(169)=1.92$, $p=0.056,95 \% \mathrm{CI}=[-0.06,5.14] ;$ neg: $\mathrm{CG}-\mathrm{UG}=-1.70, \mathrm{SE}=1.22, t(169)=-1.39, p=0.166$, $95 \% \mathrm{CI}=[-0.22,0.03])$ and arousal ratings $($ neu: $\mathrm{CG}-\mathrm{UG}=-2.07, \mathrm{SE}=1.31, t(169)=-1.58$, $p=0.117,95 \% \mathrm{CI}=[-4.67,0.52] ;$ neg: $\mathrm{CG}-\mathrm{UG}=1.86, \mathrm{SE}=1.56, t(169)=1.19, p=0.235$, $95 \% \mathrm{CI}=[-1.22,4.97])$. Thus, neither valence nor arousal models support the hypothesis of an affect intensification in the $\mathrm{UG}(\mathrm{H} 2 \mathrm{a})$ or in the $\mathrm{CG}(\mathrm{H} 2 \mathrm{~b})$ as compared to the other group. Experiment 2 exploratory models are summarized in Table 4. 


\begin{tabular}{|c|c|c|c|c|c|c|c|c|}
\hline Model & Parameter & Estimate & SE & $\mathbf{t}$ & df & $\mathbf{p}$ & & CI \\
\hline \multirow{7}{*}{$\begin{array}{l}\text { Expectancy } \\
\text { ratings }\end{array}$} & Intercept & 53.40 & 0.54 & 99.62 & 169 & $<0.001$ & 52.34 & 54.46 \\
\hline & $\mathrm{UG}-\mathrm{CG}$ & 0.63 & 1.07 & 0.59 & 169 & 0.557 & -1.48 & 2.75 \\
\hline & blue - red & -18.62 & 2.18 & -8.56 & 169 & $<0.001$ & -22.92 & -14.33 \\
\hline & color $\mathrm{x}$ group & -18.69 & 4.35 & -4.30 & 169 & $<0.001$ & -27.28 & -10.10 \\
\hline & $\sigma$ ID & 6.28 & & & & & & \\
\hline & $\sigma$ color & 27.73 & & & & & & \\
\hline & $\sigma$ residual & 19.56 & & & & & & \\
\hline \multirow{7}{*}{$\begin{array}{l}\text { Valence } \\
\text { ratings }\end{array}$} & Intercept & 41.46 & 0.41 & 101.44 & 169 & $<0.001$ & 40.65 & 42.26 \\
\hline & $\mathrm{UG}-\mathrm{CG}$ & 0.42 & 0.82 & 0.51 & 169 & 0.609 & -1.19 & 2.03 \\
\hline & neg - neu & -37.67 & 0.98 & -38.61 & 169 & $<0.001$ & -39.59 & -35.74 \\
\hline & valence $\mathrm{x}$ group & -4.24 & 1.95 & -2.17 & 169 & 0.031 & -8.09 & -0.39 \\
\hline & $\sigma$ ID & 4.45 & & & & & & \\
\hline & $\sigma$ valence & 11.30 & & & & & & \\
\hline & $\sigma$ residual & 18.59 & & & & & & \\
\hline \multirow{6}{*}{$\begin{array}{l}\text { Arousal } \\
\text { ratings }\end{array}$} & Intercept & 59.94 & 0.58 & 102.68 & 169 & $<0.001$ & 58.79 & 61.09 \\
\hline & UG - CG & -0.10 & 1.17 & -0.09 & 169 & 0.931 & -2.41 & 2.20 \\
\hline & neg - neu & 25.24 & 0.86 & 29.50 & 169 & $<0.001$ & 23.55 & 26.93 \\
\hline & valence $\mathrm{x}$ group & 3.94 & 1.71 & 2.30 & 169 & 0.022 & 0.56 & 7.32 \\
\hline & $\sigma$ ID & 7.03 & & & & & & \\
\hline & $\sigma$ valence & 9.48 & & & & & & \\
\hline
\end{tabular}


Table 3. Results of confirmatory LMMs on expectancy, valence and arousal ratings in

598 Experiment 2. For each model we reported the unstandardized regression coefficients, standard 599 errors (SE), 95\% confidence intervals (CI), and the associated $t$-test.

600

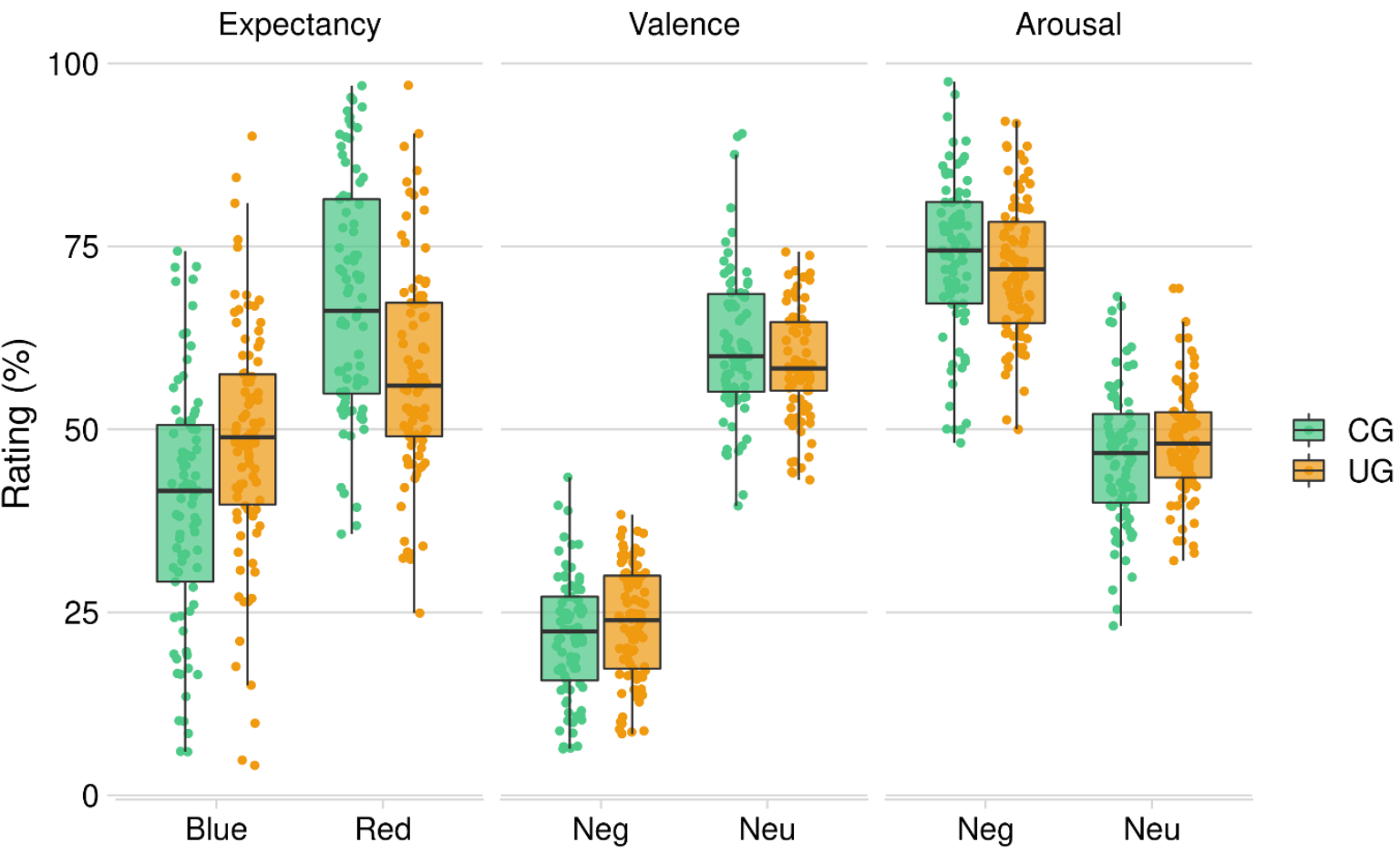

602 Figure 3. Box-plot of expectancy, valence and arousal ratings in Experiment 2 according to 603 the group (CG vs. UG), and the S1 color (red vs. blue, for expectancy ratings) or the S2 valence 604 (neg vs. neu, for valence and arousal ratings). Points represent the mean estimated value for 605 each participant and condition.

606 


\begin{tabular}{|c|c|c|c|c|c|c|c|c|}
\hline Model & Parameter & Estimate & SE & $t$ & df & $p$ & 950 & CI \\
\hline \multirow{11}{*}{$\begin{array}{l}\text { Valence } \\
\text { ratings }\end{array}$} & Intercept & 42.13 & 0.43 & 98.30 & 204.39 & $\begin{array}{c}< \\
0.001\end{array}$ & 41.29 & 42.98 \\
\hline & $\mathrm{UG}-\mathrm{CG}$ & 0.44 & 0.86 & 0.52 & 204.39 & 0.607 & -1.25 & 2.13 \\
\hline & neg - neu & -35.69 & 1.01 & $\begin{array}{c}- \\
35.36\end{array}$ & 193.50 & $\begin{array}{c}< \\
0.001\end{array}$ & $\begin{array}{c}- \\
37.68\end{array}$ & $\begin{array}{c}- \\
33.70\end{array}$ \\
\hline & congruent - incongruent & -2.70 & 0.52 & -5.22 & $6,494.00$ & $\begin{array}{c}< \\
0.001\end{array}$ & -3.71 & -1.69 \\
\hline & valence $\mathrm{x}$ group & -3.52 & 2.02 & -1.74 & 193.50 & 0.083 & -7.50 & 0.46 \\
\hline & group $\mathrm{x}$ congruency & -0.09 & 1.03 & -0.09 & $6,494.00$ & 0.929 & -2.12 & 1.93 \\
\hline & valence $\mathrm{x}$ congruency & -7.93 & 1.03 & -7.68 & $6,494.00$ & $\begin{array}{c}< \\
0.001\end{array}$ & -9.96 & -5.91 \\
\hline & $\begin{array}{l}\text { group } \mathrm{x} \text { valence } \mathrm{x} \\
\text { congruency }\end{array}$ & -2.87 & 2.07 & -1.39 & $6,494.00$ & 0.165 & -6.92 & 1.18 \\
\hline & $\sigma$ ID & 4.47 & & & & & & \\
\hline & $\sigma$ valence & 11.32 & & & & & & \\
\hline & $\sigma$ residual & 18.47 & & & & & & \\
\hline \multirow{7}{*}{$\begin{array}{l}\text { Arousal } \\
\text { ratings }\end{array}$} & Intercept & 59.48 & 0.60 & 99.45 & 186.22 & $\begin{array}{c}< \\
0.001\end{array}$ & 58.30 & 60.66 \\
\hline & $\mathrm{UG}-\mathrm{CG}$ & -0.15 & 1.20 & -0.12 & 186.22 & 0.903 & -2.51 & 2.21 \\
\hline & neg - neu & 23.85 & 0.89 & 26.67 & 201.72 & $\begin{array}{c}< \\
0.001\end{array}$ & 22.09 & 25.61 \\
\hline & congruent - incongruent & 1.83 & 0.52 & 3.52 & $6,494.00$ & $\begin{array}{c}< \\
0.001\end{array}$ & 0.81 & 2.86 \\
\hline & valence $x$ group & 3.40 & 1.79 & 1.90 & 201.72 & 0.058 & -0.12 & 6.93 \\
\hline & group $\mathrm{x}$ congruency & 0.18 & 1.04 & 0.17 & $6,494.00$ & 0.866 & -1.87 & 2.22 \\
\hline & valence $\mathrm{x}$ congruency & 5.56 & 1.04 & 5.34 & $6,494.00$ & $\begin{array}{c}< \\
0.001\end{array}$ & 3.52 & 7.60 \\
\hline
\end{tabular}




\begin{tabular}{|c|c|c|c|c|c|c|c|}
\hline $\begin{array}{l}\text { group } \mathrm{x} \text { valence } \mathrm{x} \\
\text { congruency }\end{array}$ & 2.15 & 2.08 & 1.03 & $6,494.00$ & 0.303 & -1.94 & 6.23 \\
\hline$\sigma$ ID & 7.03 & & & & & & \\
\hline$\sigma$ valence & 9.49 & & & & & & \\
\hline$\sigma$ residual & 18.62 & & & & & & \\
\hline
\end{tabular}

607 Table 4. Results of exploratory LMMs investigating the effect of S2 congruency (congruent

608 vs. incongruent) on valence and arousal ratings in Experiment 2. For each model, we reported

609 the unstandardized regression coefficients, standard errors (SE), 95\% confidence intervals (CI),

610 and the associated $t$-test.

611

612 Exploratory models comparing results of Experiment 1 and 2 are summarized in Figure

613 4. For the expectancy model $\left(\mathrm{R}^{2}\right.$ marginal $=0.184, \mathrm{R}^{2}$ conditional $\left.=0.483\right)$ we found evidence for a

614 three-way interaction $(F(1,352)=5.385, p=0.021)$ between study, group and S1 color.

615 However, none of the post-hoc comparisons between Experiment 1 and Experiment 2 emerged

616 as statistically significant. For the valence model $\left(\mathrm{R}^{2}\right.$ marginal $\left.=0.559, \mathrm{R}_{\text {conditional }}^{2}=0.645\right)$ we

617 found an interaction between study and $S 2$ valence $(F(1,352)=37.09, p<0.001)$. In particular,

618 neutral sounds were rated as more unpleasant than neutral pictures (neu: Experiment 1 -

619 Experiment $2=8.969, \mathrm{SE}=1.054, z=8.508, p<0.001)^{1}$. Finally, for arousal model $\left(\mathrm{R}^{2}\right.$ marginal

$\left.620=0.327, \mathrm{R}_{\text {conditional }}^{2} 0.512\right)$ we only found a main effect of study, with sounds rated overall as

621 more arousing than visual stimuli independently from valence (Experiment $1-$ Experiment $2=$

$622-12.3, z=-14.16, \mathrm{SE}=0.87, p<0.001)^{1}$. Thus, none of the exploratory comparisons between

623 Experiment 1 and Experiment 2 collected evidence in favor of a different effect of our

624 experimental manipulation of previous experience as a function of the sensory modality

625 involved.

\footnotetext{
${ }^{1} \mathrm{We}$ are using $z$-test for post hoc-comparison given that the computation of degrees of freedom is memory intensive due to the LMM computed on data from two experiments.
} 


\section{Experiment 1}

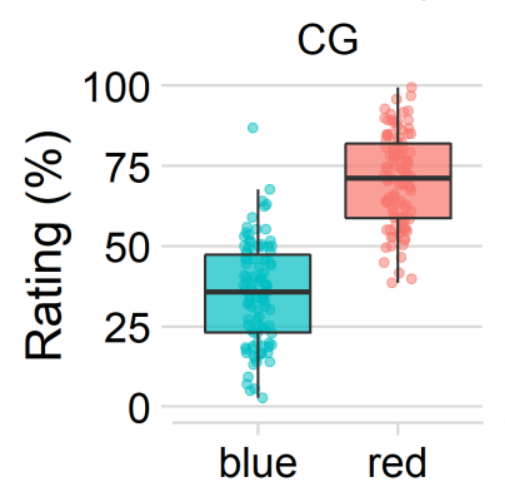

UG

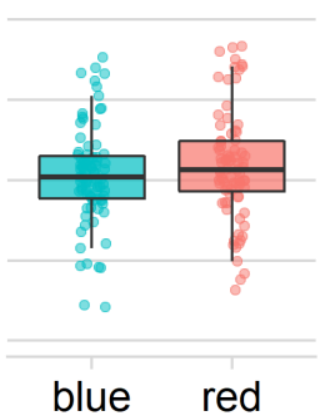

Experiment 2

CG
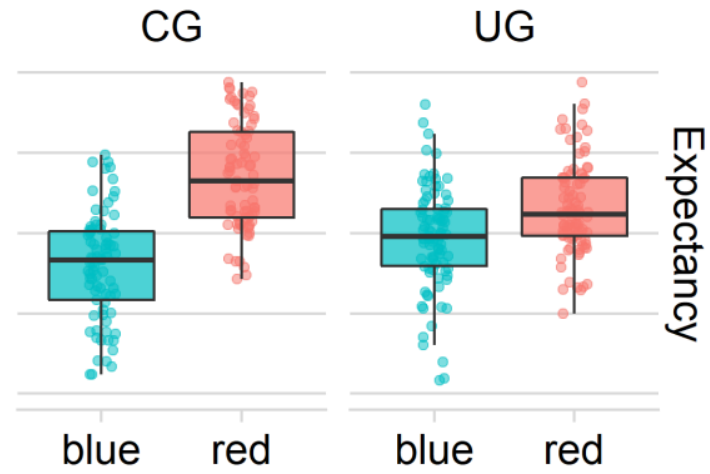
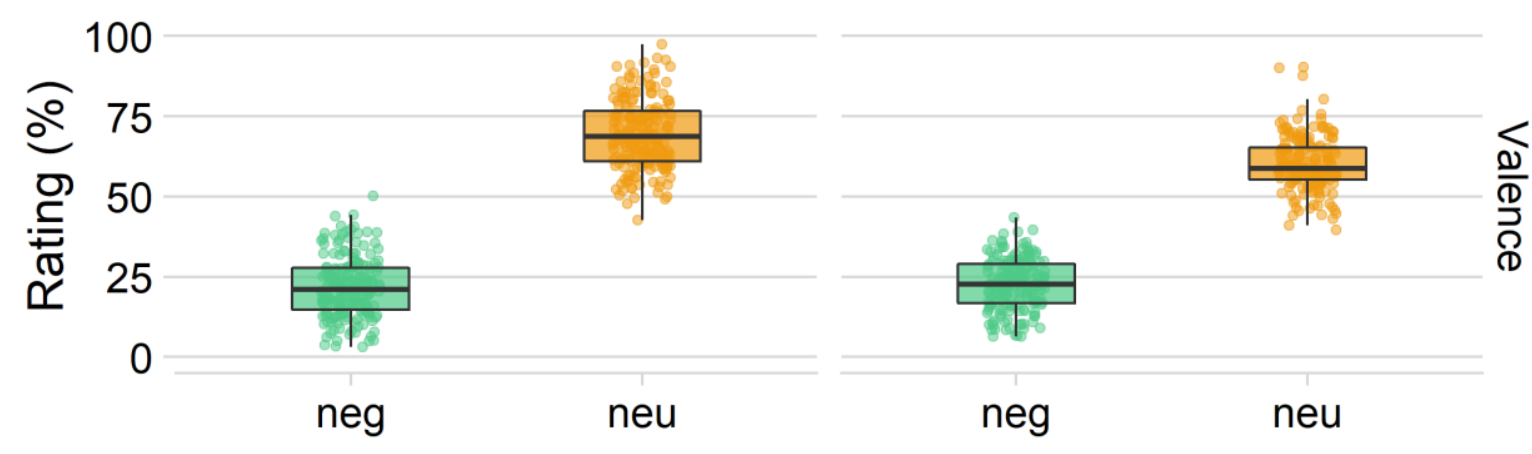

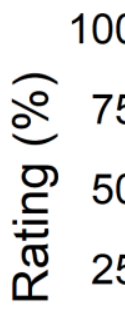

0

628 Figure 4. Top: box-plot of expectancy ratings according to the study (Experiment 1 vs.

629 Experiment 2), the group (CG vs. UG), and the S1 color (red vs. blue). Middle: box-plot of

630 valence ratings according to the study (Experiment 1 vs. Experiment 2), and S2 valence (neg

631 vs. neu). Bottom: box-plot of arousal ratings according to the study (Experiment 1 vs.

632 Experiment 2). Points represent the mean estimated value for each participant and condition. 
3.3) Discussion

Experiment 2 (visual to auditory) demonstrated that previous visual probabilistic

636 information shaped the subjective experience of the expected valence of future auditory stimuli

637 (i.e., generation-implementation stages), but not of their experienced valence and intensity (i.e., updating stage), similarly as it occurred in Experiment 1 (visual to visual). In Experiment 2 we replicated results of Experiment 1, with participants in the CG showing more negative expectancy ratings after the cues whose color was previously paired with negative stimuli.

641 Surprisingly, more negative expectancy ratings were found to follow red cues also in the UG.

642 Nevertheless, since the effect size for the red-blue contrast within the CG (-0.81) is three times 643 the effect within the UG (-0.269), this last result should be interpreted with caution. In fact, in 644 the UG the cue color had no real predictive value, because during the learning phase each color was paired equally often with either negative or neutral stimuli, thus participants could not learn any reliable S1-S2 relationship. Therefore, considering the effect sizes of both contrasts

647 and the absence of an actual predictive meaning of cues in the UG, the red-blue contrast within 648 this group may be considered of negligible significance. Overall, according to our results, experiencing a past certain environment in one sensory modality (i.e., visual) leads people's subjective expectancies to subsequently rely on previous experience to consistently generate and implement new affective predictions also in a different sensory modality (i.e., auditory). intensification effect related to previous experience. Even though we found a significant group by valence interaction in both valence and arousal models, post-hoc comparisons between groups did not reach statistical significance. Therefore, subjective reactions during the updating 
657 in a different sensory modality (i.e., visual) from the one involved in the present moment (i.e., 658 auditory).

659 Last, no evidence of a difference between sensory modalities in the influence of 660 previous experience on subjective reactions emerged. No meaningful group by study 661 interaction was found in the exploratory models run on the merged dataset (Experiment $1+$ 662 Experiment 2). Thus, consistently with the predictive framework (Barrett, 2017; Friston, 2010; 663 Seth \& Friston, 2016; Shipp, 2016), this supports the claim that affective predictions develop 664 similarly across sensory modalities. Only a general effect of sensory modality emerged with 665 regards to the updating stage, with neutral sounds eliciting more unpleasant valence ratings 666 than neutral pictures, and sounds eliciting higher arousal ratings than pictures regardless of 667 valence. These effects may be due to the heightened ambiguity of affective sounds as compared 668 to pictures (Shinkareva et al., 2014), which can produce a greater allocation of processing 669 resources towards sounds, and which can thus eventually turn into a more intense subjective 670 reaction. Alternatively, they may be ascribed to the longer presentation length of auditory 671 stimuli (i.e., $6 \mathrm{sec}$ ) as compared to visual ones (i.e., $1 \mathrm{sec}$ ).

672 
Our study represents to our knowledge the first attempt to empirically investigate the role of previous experience in the construction of new affective predictions, both within and across sensory modalities, and with a specific focus on subjective experience. We employed a novel experimental paradigm, purposely developed to address our research questions, and to

678 fill some of the gaps in the existing literature. First, our experimental design combined the logic of emotional S1-S2 paradigms with an uninstructed learning component. This allowed our paradigm to implement the role of actual previous experience in constructing new predictions, by manipulating the experienced (un)certain probabilistic relationships between stimuli. Second, it allowed us to target all the stages of the (affective) prediction construction process (generation-implementation-updating), and to study cross-modality generalization between the visual and auditory modalities. Last, by leaving participants uninstructed about the specific contingency ratios they were exposed to, our paradigm allowed us to specifically target subjective experience more similarly to how it spontaneously develops in everyday life contexts.

Overall, we found that being exposed to certain vs. uncertain previous experience affected the generation-implementation stages of future prediction construction, but not the updating stage. In both the experiments, participants who had previously experienced certain

691 probabilistic relationships between stimuli, reported more negative expectancy ratings after the 692 specific cues which were previously paired with negative stimuli. According to the predictive 693 framework (Barrett, 2017; Seth \& Friston, 2016), this supports the claim that learning certain 694 probabilistic relationships between stimuli could make the derived internal models highly 695 reliable. As a consequence, when constructing new affective predictions, subjective expectancies will fully tap into previously acquired knowledge, coherently labeling the expected valence of future stimuli. Furthermore, in line with predictive models assumptions 
698 (Barrett, 2017; Friston, 2010; Shipp, 2016), this process emerged to develop similarly (and 699 thus, to be generalizable) across the visual and auditory sensory modalities, supporting the idea 700 that (affective) predictive models are represented multimodally (Barrett, 2017; Friston, 2005; $701 \quad$ Peelen et al., 2010).

702 Surprisingly, we found no evidence about an intensification of subjective reactions 703 during the updating stage as a function of the uncertainty degrees previously experienced. 704 According to the predictive framework (Barrett, 2017; Seth \& Friston, 2016), it could be expected that having at one's disposal a reliable predictive model would lead to a pre-activation of the expected affective experience, with the consequence of producing an intensification of 707 subjective reactions when the expected stimuli actually occur. Our results, however, only 708 partially support this claim. In fact, in both the experiments we did not find any evidence of an affect intensification effect strictly related to previous experience (i.e., the two experimental groups did not significantly differ in terms of their valence and arousal ratings during the test

711 phase). However, an interesting result emerged from exploratory models assessing the 712 differences between expected and unexpected S2s by comparing affective ratings between 713 congruent and incongruent test trials (see Tables 2 and 4). In both the experiments and in both 714 the groups, congruent negative stimuli (i.e., negative stimuli more strongly expected) were 715 rated as more unpleasant and more arousing than incongruent ones (i.e., negative stimuli 716 violating expectancies), thus leading to an intensification of subjective experience. Restating 717 the differences between currently available and previously learned probabilistic information 718 (see 2.3), it is possible that experiencing a sufficient certainty level in the here and now (i.e., contingencies experienced during the test phase, $75 \%$ ) might drive subjective reactions towards an affect intensification, whereas this effect is dampened when referring to the uncertainty

721 degrees experienced in the past (i.e., contingencies experienced during the learning phase, $722100 \%$ vs. 50\%). Thus, it may be that subjective experience in the updating stage is more 
723 prominently modulated by currently available probabilistic information rather than by past learnings.

Summarizing, our results add a further piece of knowledge about the mechanisms by which previous information shapes the subjective experience of emotion, as defined within a predictive framework (Barrett, 2017; Seth \& Friston, 2016). We demonstrated that, without the need of any explicit instruction, people are able to build affective predictive models from the simple exposure to contingencies between stimuli (learning phase). This presumably happens thanks to associative learning mechanisms, which are assumed to be amongst the primary mechanisms on which prediction construction relies (Bar, 2007; Dale et al., 2012). Those affective predictive models, and their associated reliability value, are then used in a subsequent moment (test phase) to coherently predict (and make meaning of) relevant events in the present environment: the more reliable the available predictive model, the more subjective expectancies draw on it to make meaning of environmental cues and predict the emotional valence of upcoming stimuli. Furthermore, we demonstrated that this process is generalizable across sensory modalities, since the available predictive models (derived from the exposure to contingencies in the visual modality) proved to shape subjective expectancies of future stimuli also within a different (auditory) sensory modality. However, although previous experience did indeed modulate the generation-implementation of new affective predictions, it proved ineffective in shaping subjective reactions to new affective stimuli during prediction updating.

742 The actual stimuli incoming during the updating stage represent the information array that must 743 be tested against predictions, in order to adjust future predictions (Barrett, 2017; Seth \& Friston, 744 2016). Thus, in making meaning of them, it might be more crucial to draw on the information 745 available in the here and now (i.e., if the new stimuli actually confirm or violate predictions, 746 according to the present context), rather than to draw on past experience. 

Concluding, some limitations of the present study must be addressed. First, we employed unambiguous symbolic stimuli as S1s, and unimodal affective stimuli as S2s.

749 Although this choice makes our paradigm similar (and thus, comparable) with the emotional 750 S1-S2 paradigms existing in the literature, it still remains quite artificial as compared to the 751 multifaceted environments we are embedded in our everyday lives. Employing ambiguous cues, multimodal or dynamic affective stimuli, and/or different environments might allow future S1-S2 paradigms to reach a better ecological validity. Second, even though our paradigm employed more realistic (i.e., uninstructed) probabilistic ratios, participants were however invited to pay attention to the relationships between stimuli they were exposed to. Therefore, our paradigm did not allow us to understand if contingencies might be effectively learned at a fully implicit level, as it should occur according to the predictive framework (Bar, 2007). Thus, we encourage future research to overcome these limitations by employing also different kinds of affective stimuli (e.g., ambiguous, multimodal, dynamic), and by making the exposure to

760 previous experience fully implicit (e.g., by engaging participants in a distracting task during 761 the learning phase). Furthermore, as a future line of research, it could be interesting also to combine the assessment of the overt subjective experience with the measurement of more covert (psychophysiological or neural) indices. 
All materials, data and analysis code reported in this manuscript have been made

767 publicly available on OSF and can be accessed at the links https://osf.io/ef9q7/ and

768 https://osf.io/wcy9r/.

769

770

\section{6) ACKNOWLEDGEMENTS}

771

The study was supported by a grant from MIUR (Dipartimenti di Eccellenza DM

772 11/05/2017 n. 262) to the Department of General Psychology.

773

We kindly thank Filippo Carnovalini for his valuable help on the experiment

774 programming.

775 


\section{7) REFERENCES}

777

778

779

780

781

782

783

784

785

786

787

788

789

790

791

792

793

794

795

796

797

798

799

800

Bar, M. (2007). The proactive brain: Using analogies and associations to generate predictions. Trends in Cognitive Sciences, 11(7), 280-289. https://doi.org/10.1016/J.TICS.2007.05.005

Bar-Anan, Y., Wilson, T. D., \& Gilbert, D. T. (2009). The Feeling of Uncertainty Intensifies Affective Reactions. Emotion, 9(1), 123-127. https://doi.org/10.1037/a0014607

Barrett, L. F. (2017). The theory of constructed emotion: An active inference account of interoception and categorization. Social Cognitive and Affective Neuroscience, 12(1), 1-23. https://doi.org/10.1093/scan/nsw154

Barrett, L. F., Mesquita, B., Ochsner, K. N., \& Gross, J. J. (2007). The Experience of Emotion. Annual review of psychology, 58, 373-403. https://doi.org/10.1146/annurev.psych.58.110405.085709

Barrett, L. F., Wilson-Mendenhall, C. D., \& Barsalou, L. W. (2014). The conceptual act theory: A roadmap. In L. F. Barrett \& J. A. Russell (A c. Di), The psychological construction of emotion (pagg. 83-110). Guilford Press.

Bates, D., Mächler, M., Bolker, B. M., \& Walker, S. C. (2015). Fitting linear mixedeffects models using lme4. Journal of Statistical Software, 67(1). https://doi.org/10.18637/jss.v067.i01

Bermpohl, F., Pascual-Leone, A., Amedi, A., Merabet, L. B., Fregni, F., Gaab, N., Alsop, D., Schlaug, G., \& Northoff, G. (2006). Attentional modulation of emotional stimulus processing: An fMRI study using emotional expectancy. Human Brain Mapping, 27(8), 662677. https://doi.org/10.1002/hbm.20209

Bradley, M. M., \& Lang, P. J. (2007). The International Affective Digitized Sounds (2nd Edition; IADS-2): Affective ratings of sounds and instruction manual. Technical report B-3. University of Florida, Gainesville, Fl. 

uncertainty. Journal of Anxiety Disorders, 39, 30-43. https://doi.org/10.1016/j.janxdis.2016.02.007

Clark, A. (2013). Whatever next? Predictive brains, situated agents, and the future of

807 cognitive science. Behavioral and Brain Sciences, 36(3), 181-204. https://doi.org/10.1017/S0140525X12000477

Dale, R., Duran, N. D., \& Morehead, J. R. (2012). Prediction during statistical learning,

810 and implications for the implicit/explicit divide. Advances in Cognitive Psychology, 8(2), 196209. https://doi.org/10.2478/v10053-008-0115-z

813 Neural correlates of emotional predictions: A high-density EEG investigation. Brain and Cognition, 150, 105708. https://doi.org/10.1016/j.bandc.2021.105708

Dieterich, R., Endrass, T., \& Kathmann, N. (2016). Uncertainty is associated with increased selective attention and sustained stimulus processing. Cognitive, Affective, \& Behavioral Neuroscience, 16(3), 447-456. https://doi.org/10.3758/s13415-016-0405-8 of Uncertainty: A Review of the Literature and Implications for Treatment. Clinical 820 Psychology: Science and Practice, 21(3), 280-300. https://doi.org/10.1111/cpsp.12077 Friston, K. (2005). A theory of cortical responses. Philosophical transactions of the 

generalized linear mixed models by simulation. Methods in Ecology and Evolution, 7(4), 493498. https://doi.org/10.1111/2041-210X.12504

830 Anticipation of high arousal aversive and positive movie clips engages common and distinct

831 neural substrates. Social Cognitive and Affective Neuroscience, 10(4), 605-611. https://doi.org/10.1093/scan/nsu091

Grupe, D. W., \& Nitschke, J. B. (2011). Uncertainty is associated with biased

834 expectancies and heightened responses to aversion. Emotion, 11(2), 413-424. 835 https://doi.org/10.1037/a0022583

Herwig, U., Kaffenberger, T., Baumgartner, T., \& Jäncke, L. (2007). Neural correlates of a «pessimistic» attitude when anticipating events of unknown emotional valence. NeuroImage, 34(2), 848-858. https://doi.org/10.1016/j.neuroimage.2006.09.035

840 about stimulus valence on behavioural and electrophysiological responses to emotional 841 pictures. International Journal of Psychophysiology, 144, 47-55. https://doi.org/10.1016/j.ijpsycho.2019.08.002

844 Tests in Linear Mixed Effects Models. Journal of Statistical Software, 82(13), 1-26. 845 https://doi.org/10.18637/jss.v082.i13

846 Lange, K., Kühn, S., \& Filevich, E. (2015). "Just Another Tool for Online Studies" 847 (JATOS): An Easy Solution for Setup and Management of Web Servers Supporting Online 848 Studies. PLOS ONE, 10(6), e0130834. https://doi.org/10.1371/journal.pone.0130834 
850 Detect, and Manage Univariate and Multivariate Outliers, With Emphasis on Pre-Registration. on ERPs to Emotional Pictures Depend on Emotional Valence. Frontiers in Psychology, 6(1927). https://doi.org/10.3389/fpsyg.2015.01927 of Psychophysiology, 129, 41-51. https://doi.org/10.1016/j.ijpsycho.2018.04.012

Lin, H., Liang, J., Liu, T., Liang, Z., \& Jin, H. (2020). Cue Valence Influences the

Effects of Cue Uncertainty on ERP Responses to Emotional Events. Frontiers in Human Neuroscience, 14, 140. https://doi.org/10.3389/fnhum.2020.00140 enhances the P2 and P3 in their later recognition. Frontiers in Human Neuroscience, 9, 646. https://doi.org/10.3389/fnhum.2015.00646

Nencki Affective Picture System (NAPS): Introduction to a novel, standardized, wide-range, high-quality, realistic picture database. Behavior Research Methods, 46(2), 596-610. https://doi.org/10.3758/s13428-013-0379-1 
875 S2 paradigm for exploring brain mechanisms underlying affective modulation of expectancy. In Brain Mapping Research Developments (pagg. 197-209). Nova Science Publishers. Nakagawa, S., Johnson, P. C. D., \& Schielzeth, H. (2017). The coefficient of 878 determination R2 and intra-class correlation coefficient from generalized linear mixed-effects 879 models revisited and expanded. Journal of The Royal Society Interface, 14(134), 20170213. https://doi.org/10.1098/rsif.2017.0213 of Perceived Emotions in the Human Brain. Journal of Neuroscience, 30(30), 10127-10134. https://doi.org/10.1523/JNEUROSCI.2161-10.2010

Qiao, Z., Geng, H., Wang, Y., \& Li, X. (2018). Anticipation of uncertain threat 885 modulates subsequent affective responses and covariation bias. Frontiers in Psychology, 9, 2547. https://doi.org/10.3389/fpsyg.2018.02547

E. E., \& Nitschke, J. B. (2010). Uncertainty during anticipation modulates neural responses to aversion in human insula and amygdala. Cerebral Cortex, 20(4), 929-940. https://doi.org/10.1093/cercor/bhp155 M., \& Brühl, A. B. (2015). Psychophysiological responses during the anticipation of emotional

893 pictures. Journal of Psychophysiology, 29(1), 13-19. https://doi.org/10.1027/0269$894 \quad 8803 / \mathrm{a} 000129$ 
900 Shinkareva, S. V., Wang, J., Kim, J., Facciani, M. J., Baucom, L. B., \& Wedell, D. H. 901 (2014). Representations of modality-specific affective processing for visual and auditory 902 stimuli derived from functional magnetic resonance imaging data. Human Brain Mapping, 903 35(7), 3558-3568. https://doi.org/10.1002/hbm.22421

904 Shipp, S. (2016). Neural elements for predictive coding. Frontiers in Psychology, 7, 905 1792. https://doi.org/10.3389/fpsyg.2016.01792

906 Sterling, P. (2012). Allostasis: A model of predictive regulation. Physiology \& 907 Behavior, 106(1), 5-15. https://doi.org/10.1016/j.physbeh.2011.06.004

908

909 


\begin{tabular}{|c|c|}
\hline Valence & NAPS pictures names \\
\hline $\mathrm{Neg}$ & 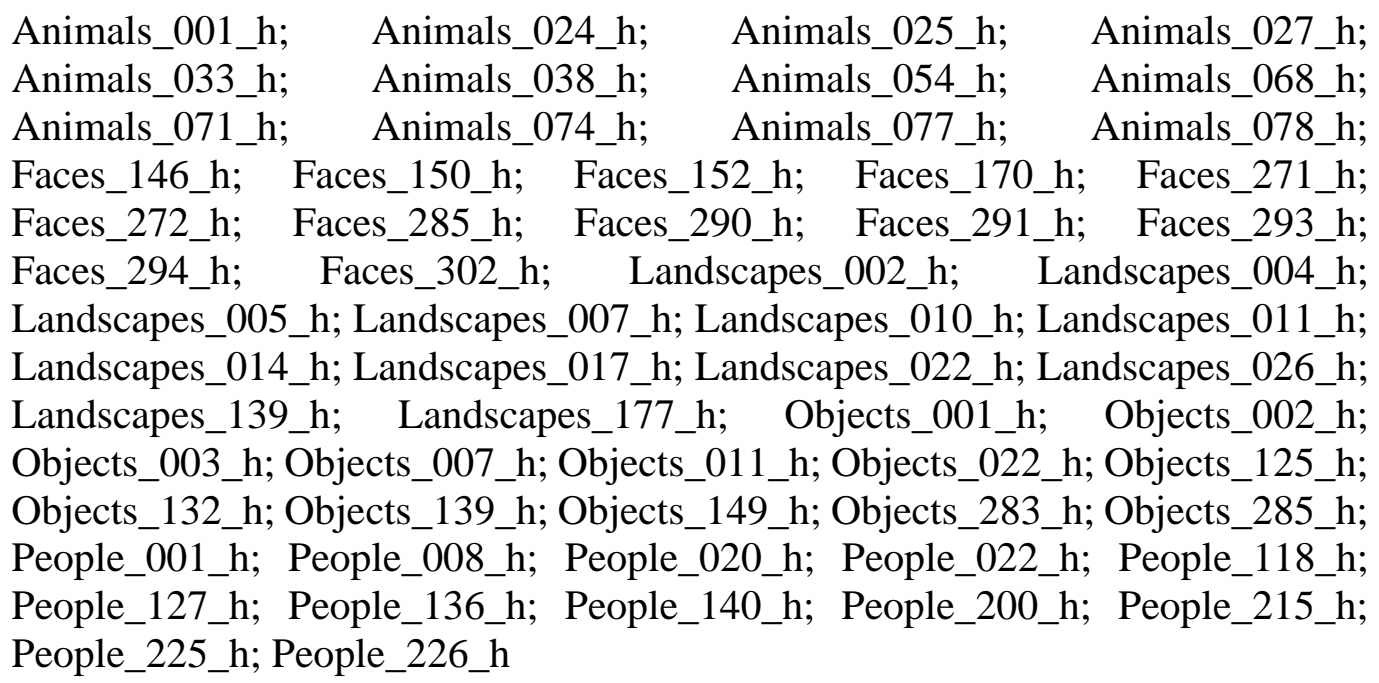 \\
\hline $\mathrm{Neu}$ & $\begin{array}{l}\text { Animals_109_h; Animals_114_h; Animals_122_h; } \\
\text { Animals_126_h; Animals_125_h; } \\
\text { Animals_170_h; Animals_136_h; Animals_165_h; } \\
\text { Faces_184_h; Faces_186_h; Faces_188_h; Faces_282_h; Faces_304_h; } \\
\text { Faces_314_h; Faces_316_h; Faces_326_h; Faces_329_h; Faces_331_h; } \\
\text { Faces_335_h; Faces_343_h; Landscapes_009_h; Landscapes_041_h; } \\
\text { Landscapes_048_h; Landscapes_050_h; Landscapes_089_h; Landscapes_100_h; } \\
\text { Landscapes_107_h; Landscapes_127_h; Landscapes_143_h; Landscapes_149_h; } \\
\text { Landscapes_163_h; Landscapes_172_h; Objects_025_h; Objects_033_h; } \\
\text { Objects_041_h; Objects_069_h; Objects_075_h; Objects_078_h; Objects_079_h; } \\
\text { Objects_103_h; Objects_254_h; Objects_262_h; Objects_263_h; Objects_270_h; } \\
\text { People_069_h; People_089_h; People_099_h; People_101_h; People_109_h; } \\
\text { People_153_h; People_162_h; People_167_h; People_173_h; People_178_h; } \\
\text { People_194_h; People_250_h }\end{array}$ \\
\hline
\end{tabular}

912 Table S1. List of NAPS picture names used as S2s in Experiment 1, sorted by valence $(\mathrm{Neg}=$

913 negative, $\mathrm{Neu}=$ neutral). 


\begin{tabular}{|c|c|c|c|c|c|c|}
\hline \multirow{2}{*}{ Measure } & \multicolumn{2}{|c|}{ Neg } & \multicolumn{2}{|c|}{ Neu } & \multirow{2}{*}{$t_{118}$} & \multirow{2}{*}{$p$} \\
\hline & $M$ & $S D$ & $M$ & $S D$ & & \\
\hline luminance & 114.149 & 27.503 & 116.943 & 27.615 & -0.555 & 0.58 \\
\hline contrast & 65.682 & 11.569 & 66.225 & 11.490 & -0.258 & 0.797 \\
\hline
\end{tabular}

complexity

\begin{tabular}{ccccccc}
\hline jpeg_size & 350012.367 & 118541.318 & 331739.683 & 120579.766 & 0.837 & 0.404 \\
\hline entropy & 7.579 & 0.338 & 7.608 & 0.311 & -0.489 & 0.626
\end{tabular}

color space

\begin{tabular}{rcccccc}
\hline LABL & 47.078 & 11.035 & 48.411 & 11.090 & -0.66 & 0.511 \\
\hline LABA & 1.684 & 4.6 & 0.541 & 7.901 & 0.969 & 0.335 \\
\hline LABB & 7.444 & 9.766 & 7.492 & 11.416 & -0.025 & 0.980 \\
\hline
\end{tabular}

915 Table S2. Means (M), standard deviations (SD), and results of two-tailed t-tests assuming

916 unequal variance in luminance, contrast, complexity indices (i.e., JPEG size, entropy), and

917 color space indices (i.e., LABL, LABA, LABB), referred to negative (Neg) and neutral (Neu)

918 NAPS pictures employed as S2s in Experiment 1.

919 
Animals_074_h, Animals_077_h, Animals_078_h, 105, 106, 115, 116, 241, Animals_024_h, Faces_293_h, Faces_290_h, 242, 244, 255, 276, 277, Faces_302_h, Faces_152_h, Landscapes_139_h, 279, 283，285，286，289, Neg Landscapes_005_h, $\quad$ Landscapes_026_h, 290, 292, 293, 295, 296, Objects_125_h, Objects_149_h, Objects_003_h, 502, 600, 611，624，625, People_226_h, People_022_h, People_140_h, 626, 703, 709, 711, 712, People_127_h 713, 714, 719, 730, 732

Animals_170_h, Animals_206_h, Animals_125_h, 107, 109, 113, 120, 132, Animals_109_h, Faces_304_h, Faces_316_h, 150, 152, 170, 171, 172, Faces_331_h, Faces_326_h, Landscapes_127_h, 206, 225, 254, 262, 270, $\mathrm{Neu}$ Landscapes_149_h, Landscapes_107_h, Landscapes_163_h, 355, 360, 361, 363, 364, Objects_262_h, 365, 370, 374, 375, 377, Objects_254_h, Objects_263_h, Objects_078_h, 378, 400, 403, 601, 602, People_194_h, People_099_h, People_173_h, 610, 698, 704, 705, 716, People_101_h 721, 724, 725, 726, 808 


\begin{tabular}{|c|c|c|c|c|c|c|}
\hline \multirow{2}{*}{ Measure } & \multicolumn{2}{|c|}{ Neg } & \multicolumn{2}{|c|}{ Neu } & \multirow{2}{*}{$t 38$} & \multirow{2}{*}{$p$} \\
\hline & $M$ & $S D$ & $M$ & $S D$ & & \\
\hline luminance & 110.232 & 23.168 & 121.242 & 24.168 & -1.471 & 0.150 \\
\hline contrast & 65.834 & 10.017 & 61.919 & 10.617 & 1.199 & 0.238 \\
\hline jpeg_size & 345480.200 & 115725.462 & 357945.550 & 111068.563 & -0.348 & 0.730 \\
\hline entropy & 7.623 & 0.399 & 7.663 & 0.216 & -0.394 & 0.695 \\
\hline LABL & 45.552 & 9.136 & 50.539 & 9.667 & -1.677 & 0.102 \\
\hline LABA & 2.089 & 4.294 & -1.576 & 10.822 & 1.408 & 0.167 \\
\hline LABB & 5.316 & 5.992 & 6.630 & 15.278 & -0.358 & 0.722 \\
\hline \multirow{2}{*}{ Measure } & \multicolumn{2}{|c|}{ Neg } & \multicolumn{2}{|c|}{ Neu } & \multirow{2}{*}{$t_{78}$} & \multirow{2}{*}{$p$} \\
\hline & $M$ & $S D$ & $M$ & $S D$ & & \\
\hline $\min \mathrm{dB}$ & -0.673 & 0.058 & -0.657 & 0.094 & -0.916 & 0.362 \\
\hline $\max \mathrm{dB}$ & 0.668 & 0.052 & 0.681 & 0.116 & -0.638 & 0.526 \\
\hline peak dB & -93.125 & 566.920 & -3.582 & 1.347 & -0.999 & 0.321 \\
\hline
\end{tabular}
unequal variance in luminance, contrast, complexity indices (i.e., JPEG size, entropy), and color space indices (i.e., LABL, LABA, LABB) for affective pictures, and in physical properties (i.e., $\min \mathrm{dB}$, max $\mathrm{dB}$, peak $\mathrm{dB}$ ) for affective sounds, referred to negative (Neg) and neutral (Neu) NAPS pictures and IADS-2 sounds employed as S2s in Experiment 2. 